\section{D) Check for updates}

Cite this: Mater. Adv., 2021, 2, 3459

Received 23rd November 2020 Accepted 4th May 2021

DOI: 10.1039/d0ma00916d

rsc.li/materials-advances

\title{
Self-assembly of carbohydrate-based small amphiphiles and their applications in pathogen inhibition and drug delivery: a review
}

\author{
Ayushi Mittal $\uparrow^{\mathrm{a}}$ Krishna, $\dagger^{\mathrm{a}}$ Aarti, ${ }^{\mathrm{a}}$ Suchita Prasad, ${ }^{\mathrm{a}}$ Prashant Kumar Mishra, (D) b \\ Sunil K. Sharma iD *a and Badri Parshad iD *c
}

\begin{abstract}
Carbohydrates are naturally occurring compounds that are biocompatible, biodegradable and offer good opportunities for chemical modifications. Also, they hold significant importance in numerous biological processes including immune response, growth regulation, cell signalling, adhesion and fertilization. Due to these characteristics carbohydrates have been successfully incorporated in amphiphilic architectures and are being used for biomedical applications such as drug delivery, gene therapy, diagnostic imaging and photosensitizer delivery. Amphiphiles self-assemble in an aqueous medium, producing myriad nanostructures with unique morphologies and properties. Herein, we review the recent developments in the area of carbohydrate-based small non-ionic amphiphiles and their self-assembly in an aqueous medium to produce various nanostructures at the molecular level. Special attention has been paid to highlight their supramolecular self-assembly behaviour and on applications of the formed selfassembled nanostructures in pathogen inhibition and drug delivery.
\end{abstract}

\section{Introduction}

Amphiphiles, described by dual affinity, self-assemble as an outcome of the interplay between hydrophobic and hydrophilic segments in an aqueous medium to produce structurally precise and functional supramolecular architectures by achieving the state of minimum free energy. ${ }^{1-5}$ Besides polymeric amphiphiles, non-polymeric small amphiphiles too have gained considerable attention owing to precise control over their molecular weight, polydispersity, and hydrophobic-lipophilic balance (HLB).$^{6-9}$ Moreover, these aggregate into well-defined constructions, instead of a mixture of various compositions. ${ }^{9}$ Self-assembly of amphiphiles produces different structures ranging from micelles, helices, fibers to tubes and vesicles. ${ }^{9-13}$ The study of amphiphilic self-assembled nanostructures is of importance because of their relevance in the field of biomedical research that includes applications as solubility enhancers, surface coating agents, imaging agents, and drug/biomolecules delivery vehicles, etc. ${ }^{14}$ The demand for the generation of specific nanostructures with precise control over the size and

\footnotetext{
${ }^{a}$ Department of Chemistry, University of Delhi, Delhi 110 007, India. E-mail: sk.sharma90@gmail.com

${ }^{b}$ School of Chemical and Process Engineering, University of Leeds, Leeds LS2 9JT, UK ${ }^{c}$ Department of Chemical Engineering and Biotechnology, University of Cambridge, Cambridge CB3 OAS, UK. E-mail: bp448@cam.ac.uk

$\dagger$ Authors contributed equally to this work.
}

morphology is increasing due to their pivotal role for the regulation of various biomedical applications such as pathogen inhibition. Vonnemann et al. investigated the size-dependent inhibition of pathogens using polysulphated gold nanoparticles. ${ }^{15}$ They found that the polysulphated gold nanoparticles of size equal and larger than the size of vesicular stomatitis virus $(>50 \mathrm{~nm})$ inhibit the virus-cell binding to a larger extent in comparison to smaller nanoparticles. Lee et al. reported the self-assembly of carbohydrate-aromatic amphiphile to nanofibers. ${ }^{16}$ The length of the nanofibers was controlled by manoeuvring the ratio of different aromatic segments of amphiphiles. The resulting nanofibers were used to systematically regulate the proliferation and agglutination of specific bacterial cells and found that the length of the nanofiber plays a critical role in bacterial cell agglutination which decreases with a decrease in length of the fibers. Several other reports have also shown that the shape, size and morphology of architecture play an important role in the inhibition of pathogenic cells. ${ }^{17-21}$ Moreover, the size of the nanostructure formed is also important to confer them drug delivery ability. The literature reports suggest that small nanocarriers of size ranging from 8-20 nm can not only easily penetrate the deep region of interstitial tissue, but also help to avoid the rapid renal clearance of the delivered drug. ${ }^{22}$ Furthermore, the membrane composed of non-ionic amphiphilic nanocarrier shields the drug from non-specific binding, partial degradation, and cellular environment until it reaches the target site, 
thus making them effective drug carriers. Depending upon the types of the hydrophilic moiety, small non-ionic amphiphiles can be categorized into different classes such as carbohydrate, peptide, polyethylene glycol (PEG), and polyglycerol-based amphiphiles and so forth. However, certain advantages associated with carbohydrate-based amphiphilic architectures such as their $\mathrm{pH}$-independent self-assembling behaviour, multiple functionalities, biocompatibility and mildness to the skin, make them potential candidates for biomedical applications. ${ }^{23}$ In the present context of the review, herein we have summarized the literature reports published in recent years on different types of carbohydrate-based small non-ionic amphiphiles along with the size and shape of their self-assembled nanostructures formed in an aqueous environment, and their applications in pathogen inhibition and drug delivery. The information is described in two sections, the first one discusses lectin recognition and pathogen inhibition potential of self-assembled nanostructures formed by small carbohydrate-based amphiphiles, while the content of the second section explores carbohydrate-based nanostructures for drug delivery applications.

\section{Carbohydrate-based amphiphiles for lectin recognition and pathogen inhibition}

Carbohydrates present on the cell surface serve as the binding site and act as 'receptor' for a large number of pathogens such as bacteria, viruses, fungi, toxins, etc. Pathogens attach themselves to the carbohydrate moiety on the cell surface during the first step of infection through multivalent interaction between carbohydrates and proteins and lead to infection. For example, the influenza virus uses its hemagglutinin glycoproteins to interact with sialic acid residue on the cell to attach to the cell surface. ${ }^{24}$ Similarly, the binding of Escherichia coli and Pseudomonas aeruginosa to epithelial cells is mediated by mannose and galactose residues, respectively. ${ }^{25-28}$ Mimicking the host cell surface by attachment of carbohydrate moieties on suitable nanostructure is an alternative to avoid infection by inhibiting the binding of the pathogen to the cell. In the recent past, nanostructures formed by carbohydrate-based small molecules have emerged as potent inhibitors of lectin and infection caused by different pathogens. ${ }^{29-36}$ The system obtained by self-assembly of small amphiphiles provides a homogeneous and controlled presentation of carbohydrates on the surface of supramolecular architecture which is hard to obtain in the case of nanostructures formed by covalent attachment of carbohydrate to a solid core, where only a statistical distribution can be achieved. Thus, these self-assembled architectures represent an ideal platform for the study of the molecular aspect of cellular interactions and providing a better understanding of the lectins.

Glycosylated amphiphiles were synthesized by grafting of $N$-acetyl-glucosamine (GlcNAc) and lactose groups onto PEGstearate moiety via copper-catalysed azide-alkyne cycloaddition (CuAAC) to produce $\mathrm{C}_{18}$ PEG-GlcNAc (1a) and $\mathrm{C}_{18}$ PEG-Lac (1b),

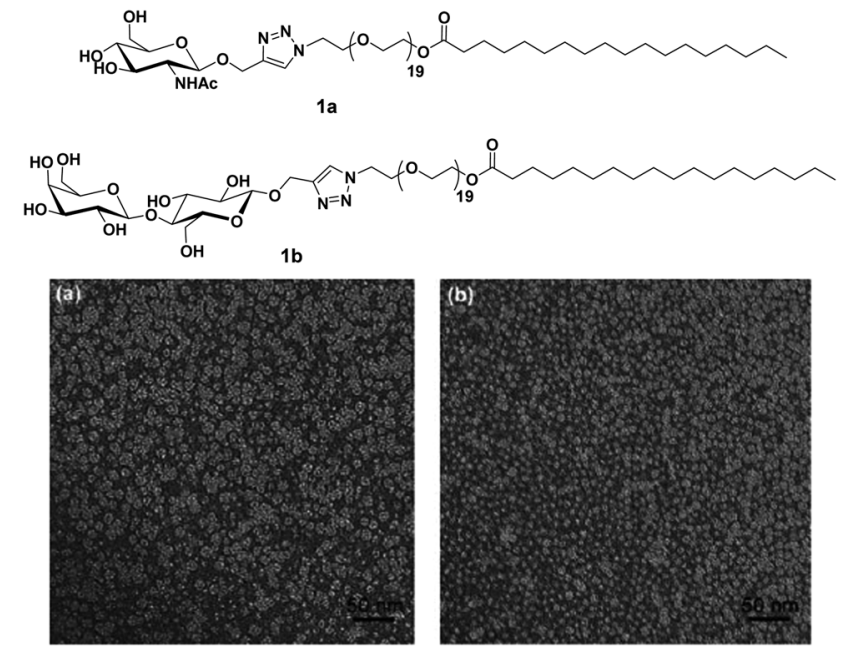

Fig. 1 Molecular structures of amphiphiles and TEM images of amphiphiles $\mathbf{1 a}$ and $\mathbf{1 b}$ showing spherical micelles of size around $10 \mathrm{~nm}$. Reprinted with permission from ref. 29. Copyright 2011 Royal Society of Chemistry.

respectively (Fig. 1). ${ }^{29}$ Stearic acid, a common $\mathrm{C}_{18}$ fatty acid, was used as a hydrophobic tail and PEG $_{900}$ (average mol. wt 900) was employed as a hydrophilic spacer to maintain hydrophilichydrophobic balance for micelle formation. Both the glycoconjugates spontaneously formed spherical micelles of size around $10 \mathrm{~nm}$ in an aqueous solution as indicated by dynamic light scattering (DLS) and transmission microscopy (TEM) studies. Sugar moieties present on the surface of micellar amphiphile 1b showed interaction with carbohydrate-binding protein Peanut agglutinin (PNA) and resulted in a significant increase of hydrodynamic radius from $10.8 \mathrm{~nm}$ to $14.8 \mathrm{~nm}$. On the other hand, non-specific lectin Wheat germ agglutinin (WGA) exhibited no affinity towards $\mathbf{1 b}$ as evident from constant hydrodynamic radius but interacted specifically with $\mathbf{1 a}$.

Furthermore, these molecules were modified with the incorporation of $\mathrm{C}_{10}$ and $\mathrm{C}_{12}$ fatty acids (2a-2d) in place of $\mathrm{C}_{18}$ along with lactose and $\mathrm{N}$-acetyl-glucosamine at the polar head of the surfactants (Fig. 2). ${ }^{30}$ DLS and small-angle X-ray scattering (SAXS) studies indicated the formation of monodisperse micelles

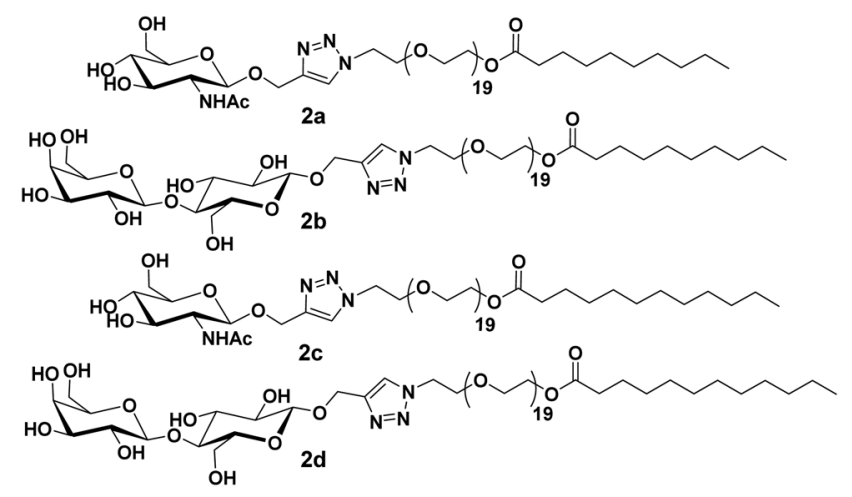

Fig. 2 Molecular structures of amphiphiles $\mathbf{2 a - 2 d}$ used for WGA lectin binding application. 
of diameter around $11 \mathrm{~nm}$. Thus, it was revealed that the size of the formed micelles is independent of the length of the fatty acid chain and the type of sugar on the surface. Furthermore, it was concluded that the hydrophilic segments may be responsible to advocate the size of micelles. WGA has a high affinity for $\beta$ - $N$-acetyl-D-glucosamine, and specifically interacted with $\mathrm{C}_{10}$ PEGGlcNAc. This was demonstrated by the DLS study, wherein a substantial increment in size to about $26 \mathrm{~nm}$ occurred, thus, confirming the presence of GlcNAc moiety on the surface of selfassembly.

Glycosylated rod amphiphiles were modified by introducing tetra( $p$-phenylene) precursor in place of stearic acid as a hydrophobic segment. PEG chain of lower size $\left(\mathrm{PEG}_{600}\right)$ was also introduced to check the effect of different hydrophilic groups (Fig. 3). ${ }^{31}$ PEG $_{600}$ based glycoconjugate self-assembled into vesicles in water with the average size distribution of $210 \mathrm{~nm}$, whereas spherical micelles were observed for glycoconjugate containing $\mathrm{PEG}_{900}$ with an average diameter of $10 \mathrm{~nm}$ as evidenced by DLS, TEM and SAXS studies. One could expect that hydrophilic/lipophilic balance is responsible to produce particles of different sizes and morphologies. Carbohydrate moieties, $N$-acetyl-glucosamine and lactose present on the surface of the micelles specifically interacted with WGA and PNA lectins, respectively, and resulted in an increase in micellar hydrodynamic radius by about $50 \%$.

Lee et al. reported the synthesis of rod-coil amphiphilic architecture consisting of tetra( $p$-phenylene) rod segment and mannose functionalized PEG as a coil (Fig. 4). ${ }^{32}$ The selfassembly of the rod-coil molecule led to the formation of nanocapsules coated with mannose units and possessed strong binding to biological receptors. They performed the role of multivalent ligands efficiently and interacted with the receptor proteins as a result of their cluster effect. This rod-coil amphiphile aggregated into stable vesicular entities with an average diameter of $36 \mathrm{~nm}$. Spherical capsules encapsulated hydrophilic fluorescent guest calcein into their interior, while their mannose-coated exterior was efficiently bound to FimH of type 1 pili bacterial cells in $E$. coli.

They further reported the formation of carbohydrate-coated nanostructures by the self-assembly of rod-coil shaped molecules that consisted of tetra( $p$-phenylene $)$ and $\operatorname{di}[\operatorname{tetra}(p$-phenylene $)]$ segments (Fig. 5). ${ }^{33}$ Increase in the PEG chain from compound 4 to compound 5a resulted in self-assembled spherical aggregates of high curvature and of approximately $20 \mathrm{~nm}$ size. Compound $\mathbf{5 b}$ consisting of a twin-rod skeleton was observed to be self-assembled

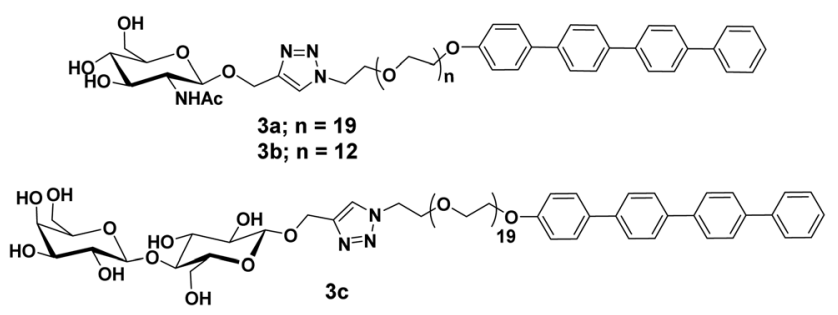

Fig. 3 Molecular structures of compounds $3 a-3 c$.

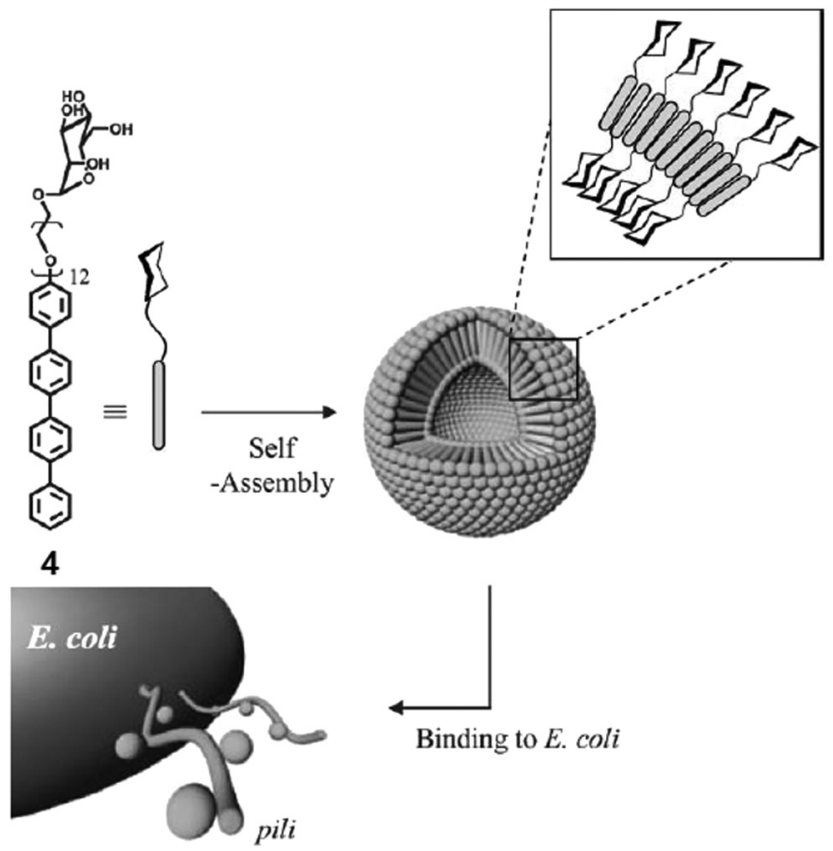

Fig. 4 Schematic representation of interaction of $E$. coli with vesicles formed by the self-assembly of mannose-functionalized amphiphile 4. Reprinted with permission from ref. 32. Copyright 2005 Royal Society of Chemistry.

into cylindrical micelles with an average diameter of $20 \mathrm{~nm}$ and having a length of several micrometres due to strong $\pi-\pi$ interaction among aromatic rings.

Nanocapsules were stabilized by the mannose unit due to carbohydrate-carbohydrate interactions and targeted the bacterial type 1 pili in $E$. coli. It demonstrated that carbohydratecoated supramolecular objects are excellent multivalent ligands. The binding ability of spherical micelles formed by compound $\mathbf{5 a}$ with Con A was evaluated by TEM imaging, where the mixture of compound 5a and Con A displayed the $180 \mathrm{~nm}$ sized spherical aggregates, much larger than the size of aggregates formed by compound 5a $(20 \mathrm{~nm})$.

The modification of rod-shaped carbohydrate-based amphiphiles was carried out by the same group by introducing an external stimuli-responsive character. They reported the synthesis, self-assembly and biological applications of the triblock aromatic dendritic skeleton. ${ }^{34}$ It consisted of flexible mannose conjugate dendron, hydrophobic alkyl chain and rigid aromatic block (Fig. 6). The formation of different supramolecular structures was observed by varying the length of the hydrophobic alkyl chain. Amphiphile 6a bearing methyl group exhibited the formation of 1D carbohydrate coated cylinders, although on increasing the length of the alkyl chain in amphiphile $\mathbf{6 b}$, the formation of the 2D lamellar structure was observed in solid state as evidenced by X-ray scatterings. However, in an aqueous solution, these rod-shaped triblock amphiphiles self-assembled into cylindrical micelles with an average diameter of $8 \mathrm{~nm}$ as indicated by DLS and TEM. The cylindrical micelles rearranged to spherical micelles upon the incorporation of the fluorescent Nile red dye and resulted in an increase of diameter to about $14 \mathrm{~nm}$. 

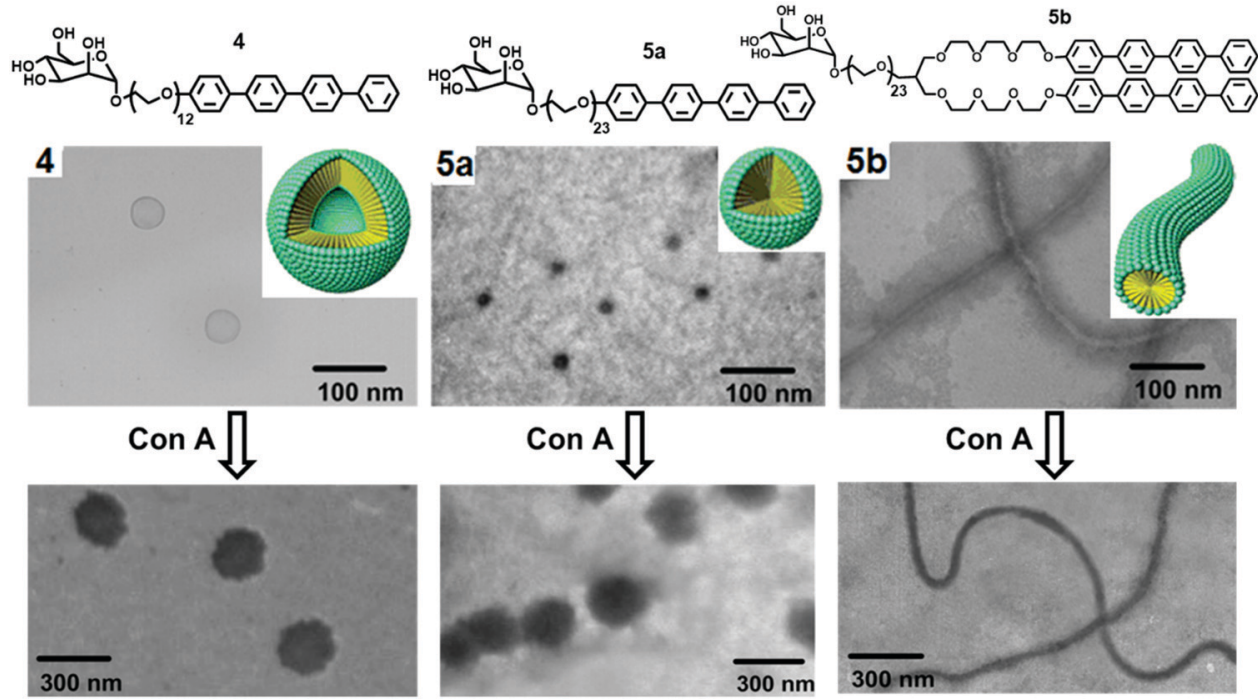

Fig. 5 Molecular structures of mannose and galactose functionalized amphiphiles $\mathbf{4 , 5 a}$ and $\mathbf{5 b}$ and schematic representation of their self-assembly into distinct nanostructures and their corresponding TEM images before and after interaction with Con A. Adapted with permission from ref. 33. Copyright 2005 American Chemical Society.

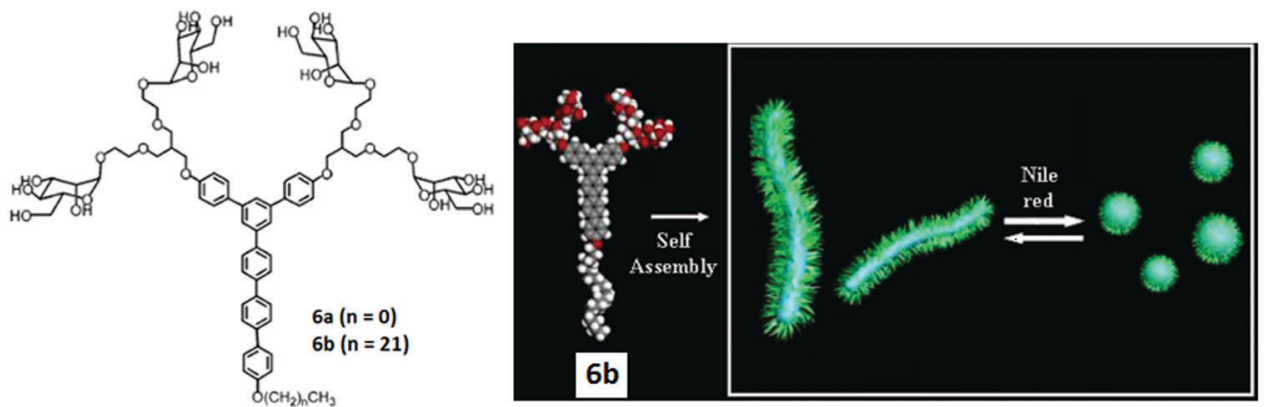

Fig. 6 Molecular structures of dendritic amphiphiles $\mathbf{6 a}$ and $\mathbf{6 b}$, and schematic representation of the reversible transformation of cylindrical micelles formed by compound $\mathbf{6 b}$ into spherical micelles in response to Nile red. Reprinted with permission from ref. 34 . Copyright 2007 American Chemical Society.

A schematic representation of the reversible transformation of cylindrical micelles formed by compound $\mathbf{6 b}$ into spherical micelles in response to Nile red is shown in Fig. 6. This was justified by the intercalation of Nile red in the aromatic core of cylindrical micelles through hydrophobic and $\pi-\pi$ interactions. Both supramolecular objects acted as a multivalent ligand for Con A, lectin and the mannose receptor on E. coli. Fluorescent labelled Con A interacted with mannose residues on the nanostructures and resulted in the precipitation of the mixture. Similarly, both cylindrical and spherical micelles responded to the bacterial pili in E. coli (ORN 178). The mannose-binding proteins (MBPs) located on the bacterial pili showed strong affinity towards mannose-coated objects and demonstrated strong multivalent interactions, but were not able to disrupt the shape and size of the objects. In addition, the shape of the nanostructures controlled the motility inhibition of E. coli.

Carbohydrate-based rod-coil amphiphilic molecules $7 \mathbf{a}$ and $7 \mathbf{b}$ containing hydrophobic aromatic segments and hydrophilic mannose moieties were reported by Lee et al. (Fig. 7). ${ }^{35}$
Molecules $7 \mathbf{a}$ (pyrene unit, highly crystalline aromatic core) and $\mathbf{7 b}$ (pentaphenylene unit, less crystalline aromatic core) having a different aromatic core self-assembled into nanofibers. The formation of self-assembled nanostructures was first investigated by TEM, which showed the generation of long, rigid nanofibers with a uniform width of $6 \mathrm{~nm}$ and length $>2 \mu \mathrm{m}$ in case of amphiphile $7 \mathrm{a}$, and short fibers with an average size of $70 \mathrm{~nm}$ for amphiphile $7 \mathbf{b}$. The co-assembly of these two nanofibers resulted in the formation of nanofibers with different length where the length of nanofiber systematically decreased from a few micrometers to less than $70 \mathrm{~nm}$ as the amount of amphiphile $\mathbf{7 b}$ increased in the co-assembled mixture of amphiphiles $7 \mathbf{a}$ and $7 \mathbf{b}$ (Fig. 7). It was found that by varying the ratio of amphiphiles $\mathbf{7 a}$ and $\mathbf{7 b}$ in their mixture and thus varying the crystallinity of aromatic cores, the length of the self-assembled nanostructure can be controlled.

Investigation of multivalent interactions between nanofibers and $E$. coli showed that cluster size decreased with a decrease in fiber length and no bacterial agglutination was observed with 


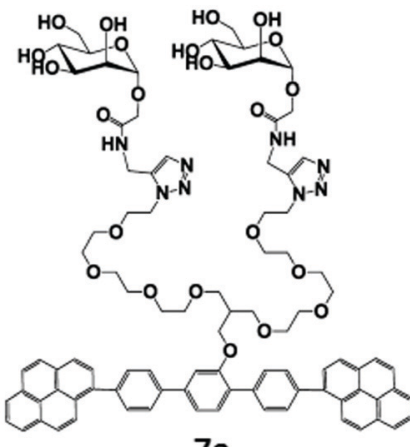

7a
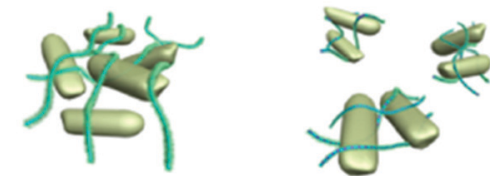

(Increasing the content of amphiphile $\mathbf{7 b}$ )

Bacterial agglutination decreases with decrease in length of fibers

Fig. 7 Molecular structures of amphiphiles $7 \mathrm{a}$ and $7 \mathrm{~b}$ and schematic representation of the regulation of agglutination and proliferation of bacterial cells by the variation of nanofiber length, where the fibre length decreases with decrease in the content of amphiphile $\mathbf{7 b}$ in a mixture of amphiphiles $\mathbf{7 a}$ and $\mathbf{7 b}$. Reprinted with permission from ref. 35. Copyright 2012 American Chemical Society.

compound $\mathbf{7 b}$. The long nanofiber was able to aggregate $E$. coli cells and formed large bacterial clusters, indicating that the length of mannose nanofiber plays a critical role in the agglutination of bacterial cells.

A similar type of carbohydrate nanofibers that displayed thermal responsive switching between binding and nonbinding states of specific bacterial cells were also reported. ${ }^{36}$ These nanofibers comprised mannose and thermo-responsive oligoether dendron-based rod-shaped amphiphiles $\mathbf{8 a}$ and $\mathbf{8 b}$ (Fig. 8). TEM studies demonstrated that both the amphiphiles self-assembled into nanofibers with an average diameter of $6 \mathrm{~nm}$. The effect of carbohydrate density on bacterial cell clustering was investigated by varying the ratio of dendron amphiphile $\mathbf{8 b}$ from 10 to $90 \mathrm{~mol} \%$ in co-assembled nanofibers. The incubation of $E$. coli with co-assembled nanofibers showed that above $70 \%$ of amphiphile $\mathbf{8 b}$, no bacterial cluster formation occurred. Thus, it was concluded that the incorporation of selective ligand at the surface of self-assembled nanostructures produce switchable nanomaterials to control, sophisticated biological functions such as signal transduction and protein inhibitions.

In recent years, $\pi$-conjugated building blocks, such as perylene bisimides have attracted special interest in the construction of nano supramolecular objects. ${ }^{37,38}$ Derivatives of perylene bisimides have already been explored in liquid crystals, organogels and organic photovoltaic devices. Wei and coworkers reported the synthesis of chiral water soluble perylene bisimides known as PBI-Lac by incorporation of a natural chiral compound, $\beta$-D-lactose, and showed its aggregation behavior and application in biology. ${ }^{37}$
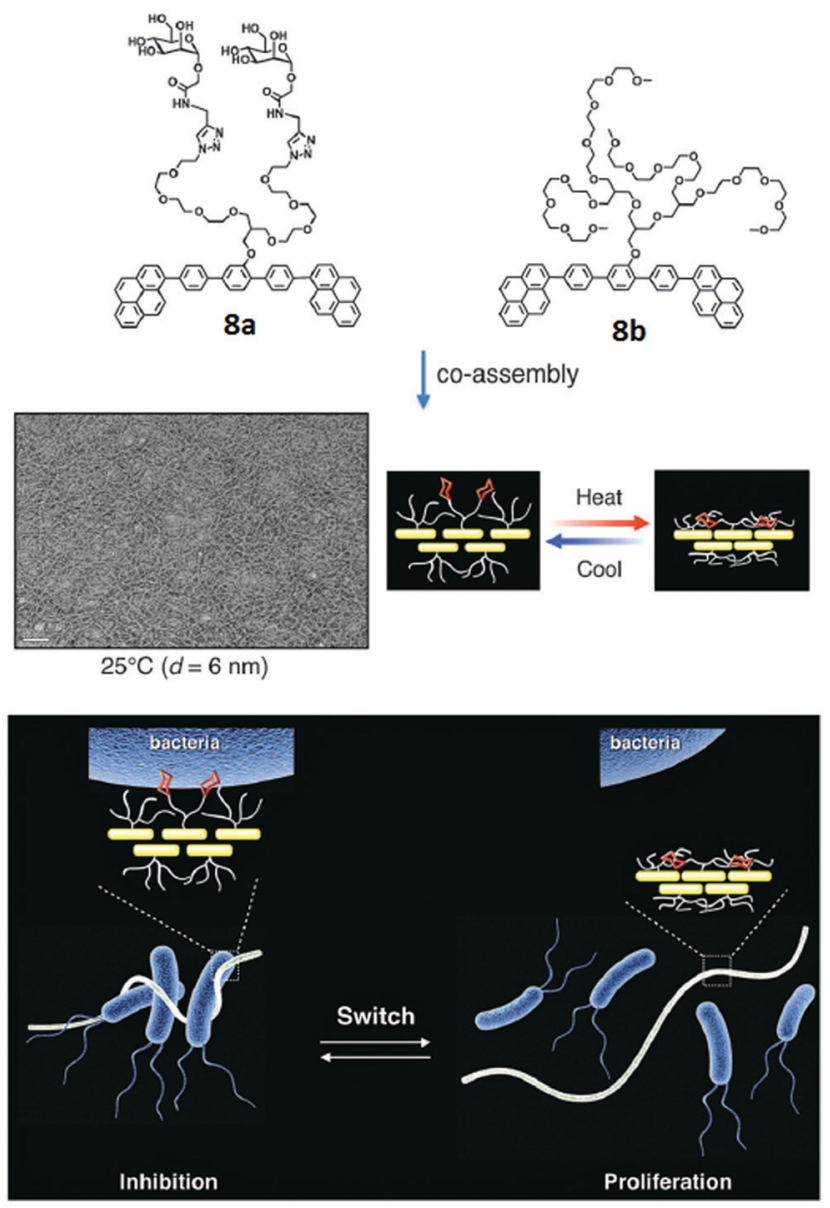

Fig. 8 Molecular structures of amphiphiles $\mathbf{8} \mathbf{a}$ and $\mathbf{8} \mathbf{b}$, and TEM image of their co-assembled nanofibers. Schematic representation of switching of carbohydrate nanofibers in binding and releasing bacteria. Reprinted with permission from ref. 36. Copyright 2016 Royal Society of Chemistry.

PBI-Lac existed in a non-aggregated state in DMSO, and three absorption bands appeared in the range of 450-550 $\mathrm{nm}$. Upon the addition of water, a new shoulder in UV-vis spectra was observed at about $554 \mathrm{~nm}$ due to the $\pi-\pi$ aggregation. Furthermore, in Circular Dichroism (CD) spectroscopy, a bisignate signal was observed, which recommended that PBI-Lac acquired right-handed supramolecular stacking. PBI-Lac self-assembled into lactose-coated supramolecular glycoclusters and interacted with PNA lectin as confirmed by turbidity assay, CD spectroscopy and cryogenic scanning electron microscopy (cryo-SEM). On addition of the PNA to PBI-Lac, the solution became turbid and showed a weak change in the CD spectrum, thus demonstrated that multivalent glycoclusters specifically bound to the PNA lectin. In addition, cryo-SEM images of PBI-Lac-PNA showed rough nanofibers with a diameter of $30 \mathrm{~nm}$. Carbohydrate-lectin interaction with PNA is shown diagrammatically in Fig. 9.

Linear nanofibers have many applications in biomedicine and material chemistry. A water-soluble scaffold (compound 10) based on perylene bisimide modified with six chiral D-mannose units was synthesized, which organized into supramolecular stacks in water due to $\pi-\pi$ stacking between backbones (Fig. 10). ${ }^{38}$ Its self-assembly depended on the percentage of 


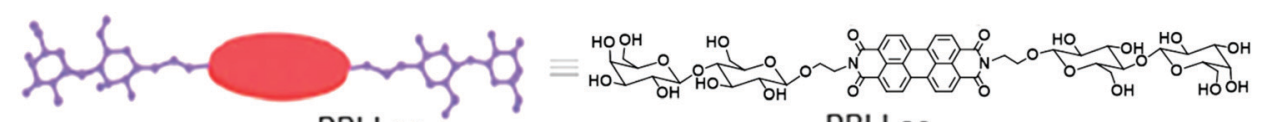

PBI-Lac

PBI-Lac
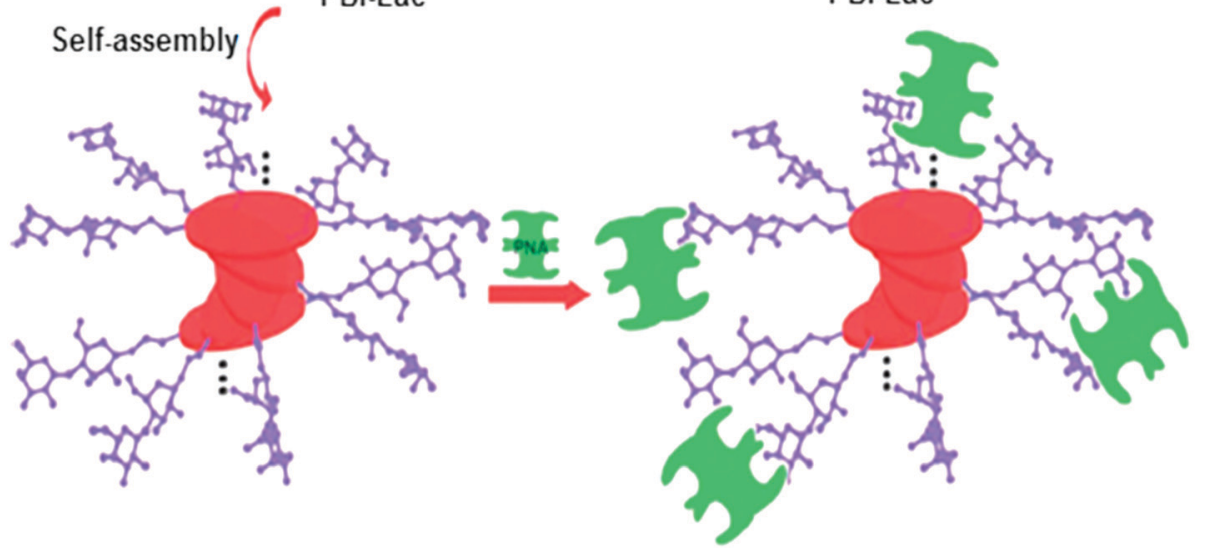

Fig. 9 Schematic representation of possible self-assembly mode of PBI-Lac and interaction with PNA lectin. Reprinted with permission from ref. 37. Copyright 2012 Royal Society of Chemistry.
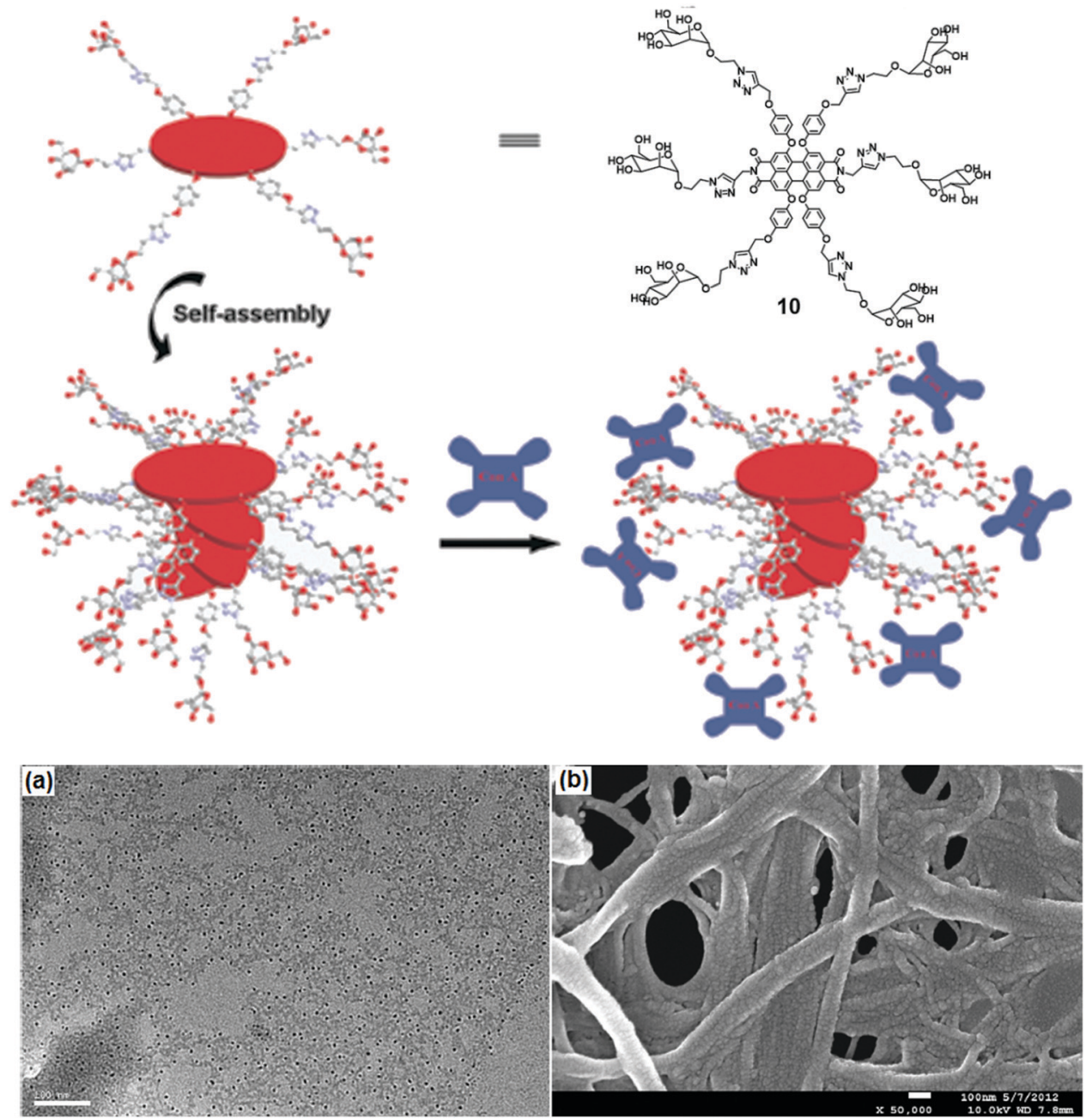

Fig. 10 Schematic representation of possible self-assembly mode of compound 10 and interaction with Con A. (a) TEM and (b) SEM images of the complexes formed between the self-assembled structure of compound 10 and Con A. Reprinted with permission from ref. 38 . Copyright 2013 Royal Society of Chemistry. 
water content in DMSO. 60\% of water taken in DMSO solution resulted in left-handed helical conformation, while an increase in the volume of water beyond $85 \%$ led to the formation of right-handed helical conformation. These mannose-based nanostructure selectively interacted with Con A as examined by turbidity assay and CD spectra. The morphology of the selfassembled nanofibers with Con A was investigated by TEM and SEM images. TEM studies revealed the formation of pellets with a diameter of $10 \mathrm{~nm}$ along with nanofibers whereas, in SEM, it was observed that Con A aggregated to nanoballs. ELLA (enzyme-linked lectin assay) experiment was used to quantify multivalent binding between glycoclusters and lectin, suggested strong binding with an $\mathrm{IC}_{50}$ value of $24 \mu \mathrm{M}$. $^{38}$

Furthermore, Lee et al. reported carbohydrate-coated $\beta$-sheet nanoribbon to control the degree of bacterial agglutination and motility. ${ }^{39}$ The triblock molecules composed of $\beta$-sheet-forming peptide and carbohydrate (mannose or glucose) attached with a PEG spacer, were employed for this purpose (Fig. 11). The selfassembly of the molecules determined by CD spectroscopy revealed that all these molecules formed $\beta$-sheets, with TEM study indicated the formation of nanoribbon. Furthermore, it was observed that the length of the nanoribbon increased with an increase in PEG chain length, suggested that PEG length played an important role in the stabilization of nanoribbon formed as well as in controlling their length. The studies also concluded that $E$. coli bacterial motility inhibition relied on the stabilisation and the length of nanoribbons. A better inhibition of bacterial motility was caused by a stable and longer nanoribbon, which may be due to the ability of longer PEG chains to precisely arrange mannose units on the FimH protein of E. coli. In addition, the specificity of mannose-coated nanoribbons towards $E$. coli was investigated by the encapsulation of fluorescent dye Nile red and it also revealed the enhancement in the sensitivity of bacterial detection due to cluster formation.

In another report, Lee et al. synthesized similar peptidebased block molecules that consisted of functional carbohydrate blocks, a coil, and a $\beta$-sheet peptide block. The resulting nanostructures displayed multiple mannose ligands on the surfaces. ${ }^{40}$ Molecules with a relatively small or linear (12a) and bulky dendritic (12b) coil block formed long and short nanostructures, respectively (Fig. 12). Due to the steric crowding effect, the bulkiness of the coils affected the final size and morphology of the assembled $\beta$-sheet peptide nanostructures. The spectroscopic and microscopic data showed that peptide molecules selfassembled into $1 \mathrm{D}$ or discrete ribbon-like aggregate with lengths of several micrometers (small and linear coil, 12a) and nanometers (bulky dendritic coil, 12b), wherein the inner core of the nanoribbons was formed of bilayered $\beta$-sheet peptide domains and the outer edge contained mannoses and triethylene glycol segments. Within the core, the peptide segments were stacked perpendicular to the long ribbon axis with an antiparallel $\beta$-sheet arrangement. The interaction of these carbohydrate-coated peptide-based nanostructures with $E$. coli was investigated, and the result showed that both the nanoassemblies could immobilize or aggregate bacterial cells. The degree of immobilization was similar for both nanoassemblies, but only long nanoribbon was found to induce the formation of bacterial clusters (Fig. 12).

Vesicles have been extensively studied due to their advanced application in the pharmaceutical industry and as biomembranes. The surface of the vesicles functionalized with natural or synthetic carbohydrate moieties results in an enhancement of its specificity towards lectins, which fulfils the fundamental criteria of specificity in therapeutic applications. In this context, Santos et al. reported self-assembled vesicles composed of phosphatidylcholine (13) and glycosylated polymeric amphiphile $N$-acetyl- $\beta$-D-glucosaminyl-PEG ${ }_{900}$-docosanate $\quad\left(\mathrm{C}_{22} \mathrm{PEG}_{900} \mathrm{GlcNAc}\right)$ as shown in Fig. 13. ${ }^{41}$ Since the surface of vesicles was occupied with $N$-acetyl-D-glucosaminyl units, they possessed the potential to bind with Bauhinia variegata lectin (BVL).

Structurally, the hydrodynamic dimension of vesicles $(\mathbf{1 3}+$ $\mathrm{C}_{22} \mathrm{PEG}_{900} \mathrm{GlcNAc}$ ) was found to be approximately $100 \mathrm{~nm}$, whereas, in the presence of lectin, the size of the assemblies increased to $130 \mathrm{~nm}$, which indicated that glycosylated surface recognized BVL lectin successfully. This morphology was further supported by SAXS and atomic force microscopy (AFM) techniques. In addition, FT-IR indicated the variation observed in the orientation of choline and the order of lipid polar head groups, and their packing after the interaction of vesicles with the lectin (BVL). These results showed that carbohydrate-coated vesicles can act as a specific receptor in a targeted nanocarrier system.

A library of amphiphilic Janus glycodendrimers was synthesized using galactose, mannose and lactose in the hydrophilic part, and exhibited three different topologies formed from

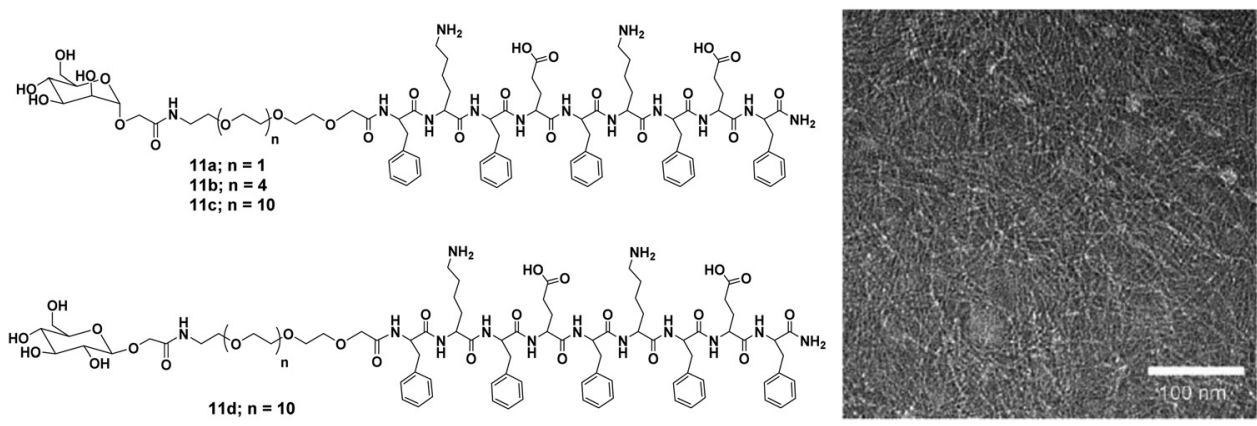

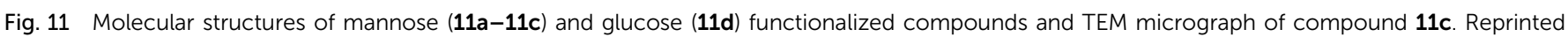
with permission from ref. 39. Copyright 2007 Wiley-VCH Verlag GmbH \& Co. KGaA. 

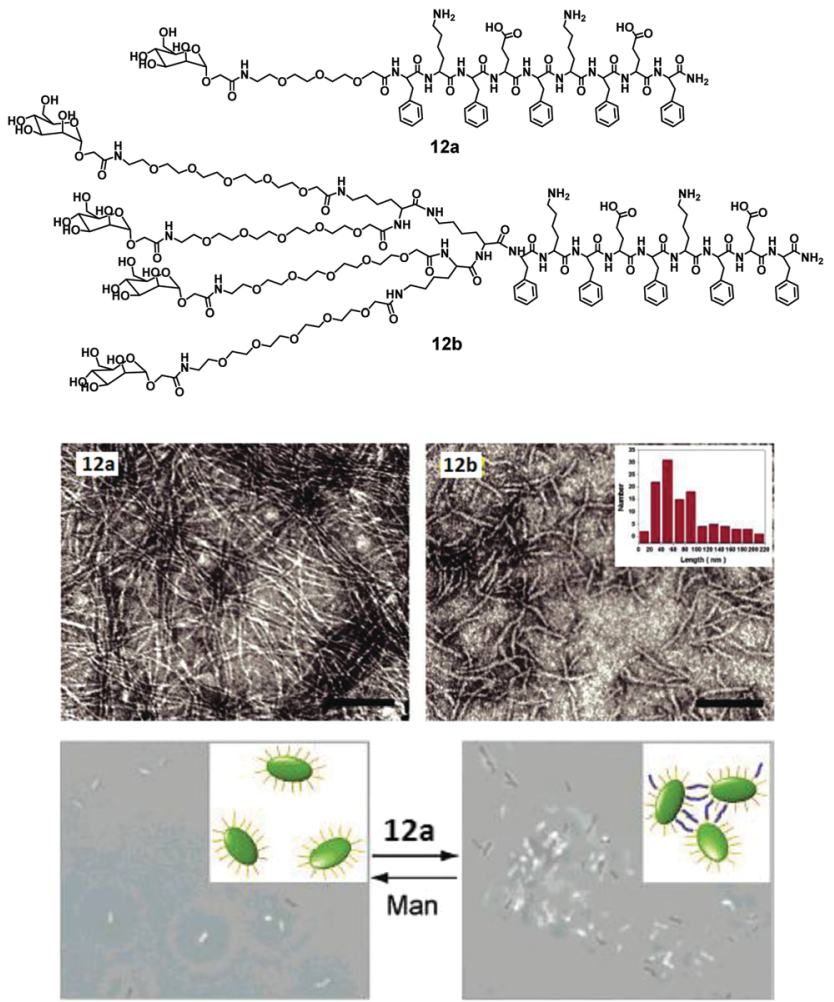

Fig. 12 Chemical structures of amphiphiles $12 \mathrm{a}$ and $\mathbf{1 2 b}$, their negatively stained TEM micrographs showing formation of nanoribbons and phasecontrast microscopic images of bacterial agglutination, where addition of amphiphile 12a to $E$. coli ORN178 resulted in motility inhibition and agglutination. Reprinted with permission from ref. 40. Copyright 2007 American Chemical Society.

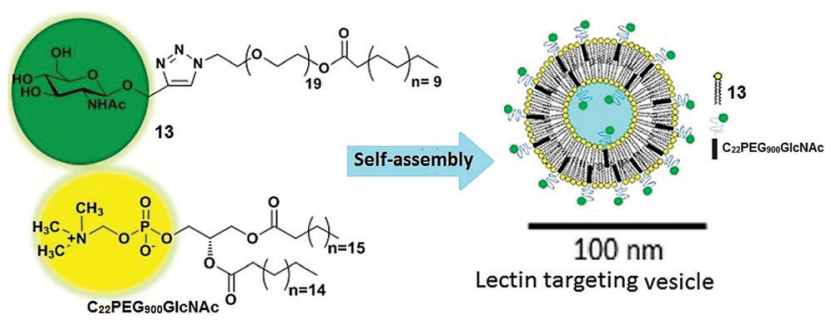

Fig. 13 Schematic representation of self-assembly of phosphatidylcholine (13) and glycosylated polymeric amphiphile ( $\left.\mathrm{C}_{22} \mathrm{PEG}_{900} \mathrm{GlcNAc}\right)$ into vesicles. Reprinted with permission from ref. 41. Copyright 2016 Elsevier.

either one or two carbohydrate head groups or a mixed conformation with a non-carbohydrate hydrophilic arm. Some representative structures of amphiphiles $(\mathbf{1 4 a - 1 4 c )}$ are shown in Fig. 14. ${ }^{42,43}$ The synthesized Janus glycodendrimers selfassembled into stable and uniform supramolecular nanostructures by simple injection of THF solutions of the dendrimers into water or buffer. Different nanostructures such as vesicles, micelles and lamellae were obtained depending upon the structure of dendrimers and the way of solution injection, where the thickness of nanostructure's membrane was found to be almost equal to the thickness of two Janus dendrimers.

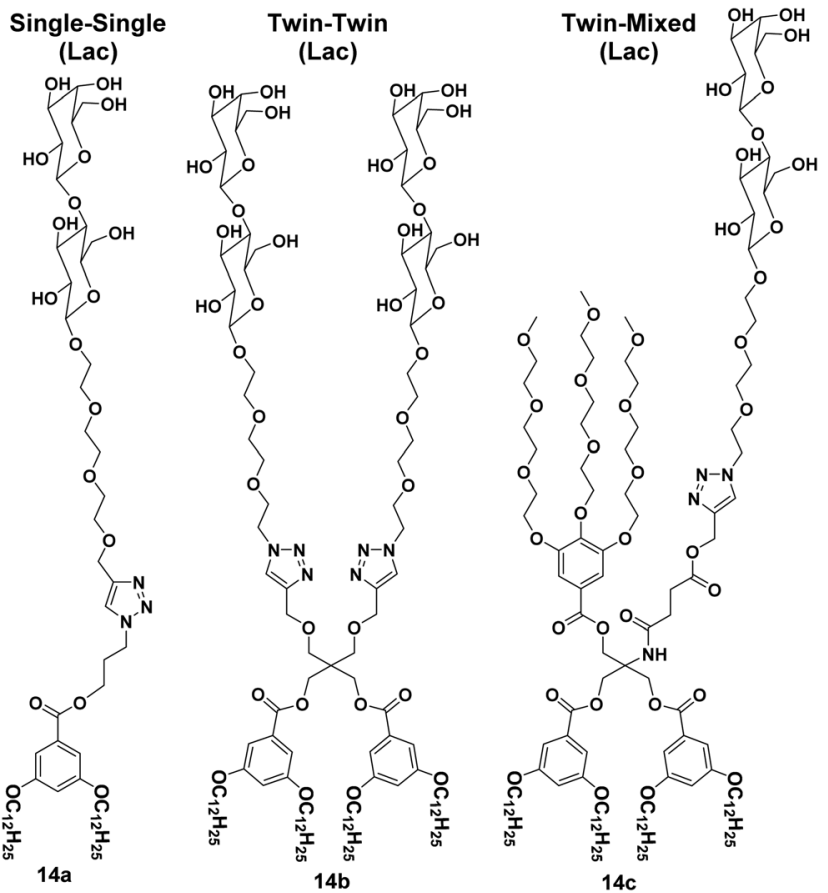

Fig. 14 Some representative structures of Janus glycodendrimers $(\mathbf{1 4 a - 1 4 c )}$ with different topologies i.e. either one or two carbohydrate head groups or a mixed conformation with a non-carbohydrate hydrophilic arm.

Further, it was observed that the interaction and agglutination of these nanostructures with lectin was affected by the amount of the sugar displayed on the surface together with the surface morphology or topological confirmation of the aggregates formed.

Glycocalixarene-based amphiphilic nanostructures have also been reported for lectin recognition and pathogen inhibition applications. The size, valency, conformation and type of surface carbohydrate groups determine their self-assembly and lectin/pathogen binding potential. Sgarlata et al. reported the synthesis of calixarene-based glycoconjugates (15a-15f, Fig. 15) using calix[4]arene and calix[8]arene as core and GlcNAc as

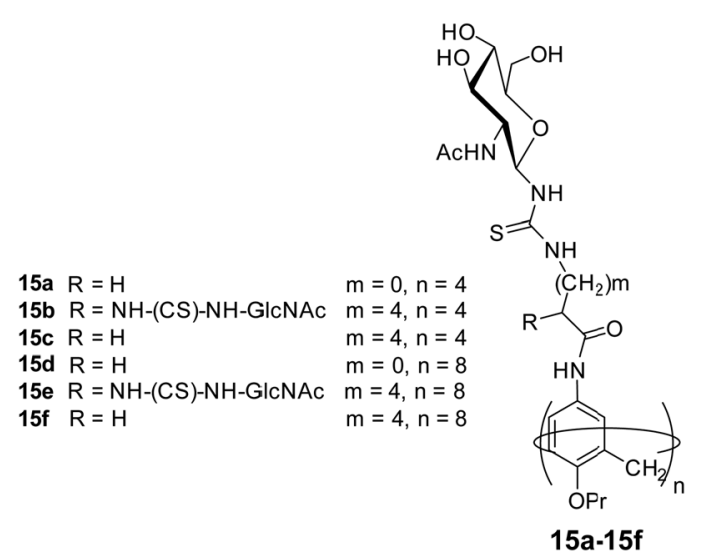

Fig. 15 Molecular structures of the glycocalixarenes (15a-15f) used to explore WGA lectin binding ability. 
surface carbohydrate unit, and investigated the activity of formed nanostructures for lectin binding by varying the nature of scaffold and spacer. ${ }^{44}$ WGA lectin was added to the solution of nanostructures, wherein a rapid formation of turbid suspension indicated the binding of WGA lectin to the glycocalixarene. These calixarenes-GlcNAc did not show any interaction with galactose specific PNA lectin which confirmed their lectin recognition specificity. Introduction of linear spacer (amino acid) between carbohydrate unit and the calixarene scaffold enhanced their lectin binding ability. Furthermore, the lectin binding ability was found to be sensitive towards the nature of the calixarene scaffold. The calix[4]arene skeleton had higher inhibition potential than conformationally mobile calix[8]arene.

Ungaro et al. reported glycocalixarenes that consisted of glucose units at the upper rim of calix[6] arene (16a) and calix[8]arene (16b), whereas the lower rim was occupied with the methyl groups (Fig. 16). ${ }^{45}$ Both macrocycles of concentration $5 \times 10^{-4} \mathrm{M}$ self-assembled in aqueous solution and formed small discoid like particles of size 10-15 $\mathrm{nm}$ as measured by the AFM experiments. DLS study also confirmed the formation of small-sized aggregates of an approximate diameter of 3-5 $\mathrm{nm}$. Furthermore, in the presence of glucoside specific Con A lectin, both the macrocycles agglutinated the lectin which was confirmed by an increase in absorbance of the solution and aggregate size.

Glucose functionalized calix[4]arene-based bolaamphiphile (17) has been incorporated into the lipid bilayer of liposome for multivalent lectin recognition (Fig. 17). ${ }^{46}$ The reported glucocalixarene bolaamphiphile restricted the flexibility of the bilayer formed by unsaturated lipids and produced the glycosylated liposomes. The addition of bolaamphiphile to fluorescence labelled Con A showed no effect on fluorescence intensity of Con A protein, but if it was used with lipid bilayer, then the intensity of labelled Con A reduced significantly, which confirmed that the glucocalixarene functionalized lipid bilayer showed specific multivalent interaction with the glucose binding protein Con A.

Huang et al. synthesized amphiphilic pillar[5]arene (18) containing galactose and alkyl chain as the hydrophilic and hydrophobic parts, respectively (Fig. 18). ${ }^{47}$ The synthesized macrocyclic amphiphile self-assembled into vesicles with an average diameter of $170 \mathrm{~nm}$ in water and transformed into nanotube after standing for one week. The existence of galactose on amphiphilic nanotubes provided multivalent ligands that had higher agglutination ability for carbohydrate receptors on $E$. coli.

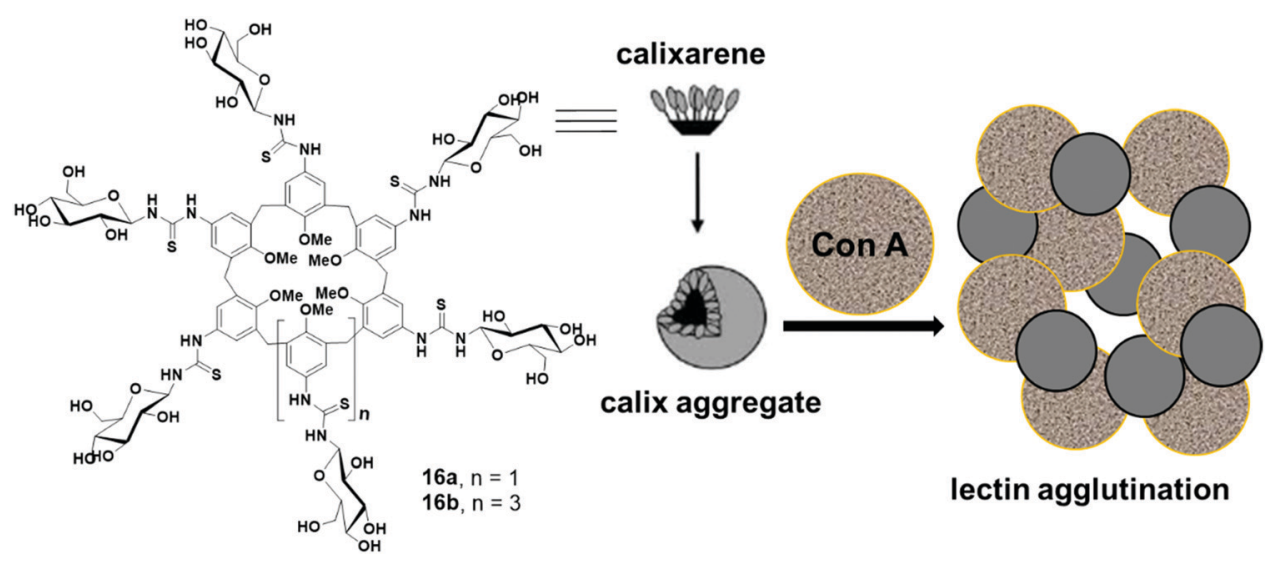

Fig. 16 Schematic representation of the aggregation processes involving glucocalixarene and Con A.
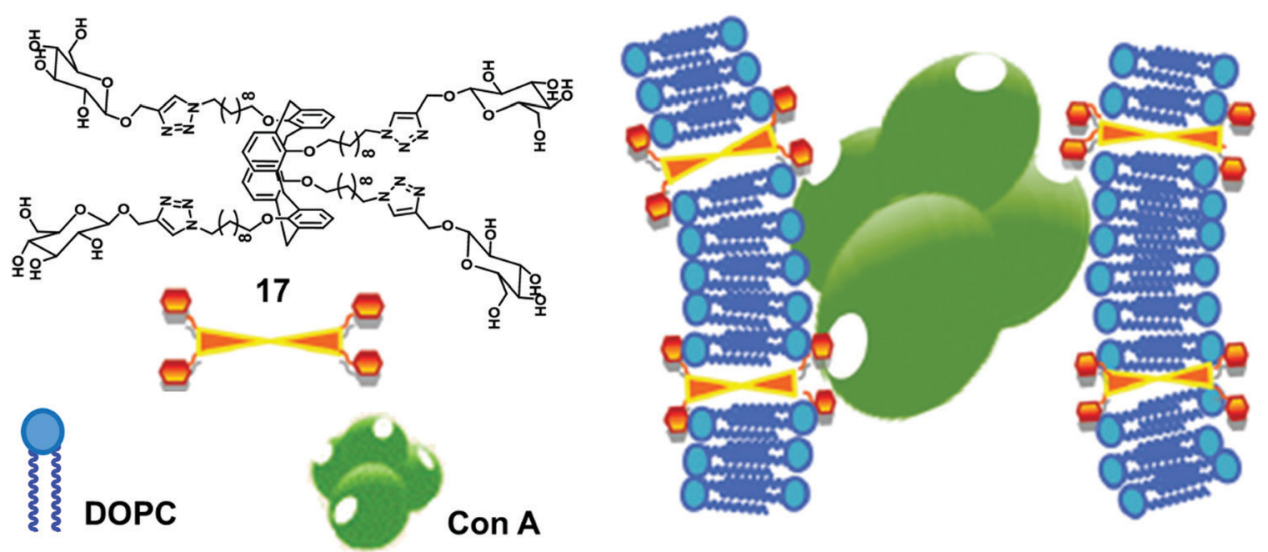

Fig. 17 Illustration of interaction of the glucosyl units of bolaamphiphile 17 incorporated in lipid bilayer with Con A. Adapted from ref. 46 with permission from The Royal Society of Chemistry. 


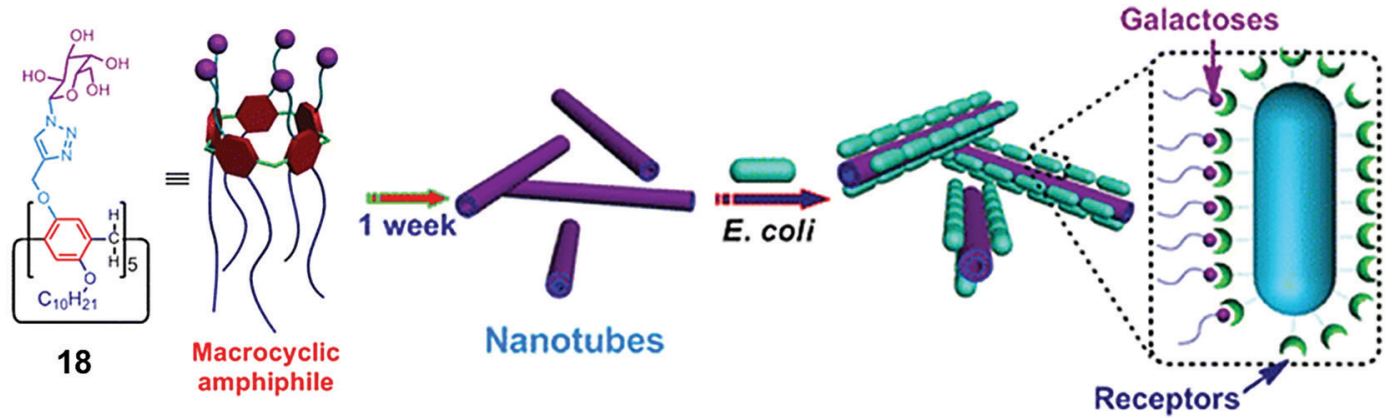

Fig. 18 Molecular structure of pillar[5]arene amphiphile (18) and schematic representation of its transformation into nanotubes and interaction with E. coli. Reprinted with permission from ref. 47. Copyright 2013 American Chemical Society.

\section{Carbohydrate-based amphiphiles as drug/dye nanocarriers}

Carbohydrate-based amphiphilic architectures have been successfully synthesized and investigated as advanced nanocarriers for drug delivery applications. ${ }^{48,49}$ Carbohydrates, exhibiting a well-defined polyhydroxy architecture whose hydroxyl groups can be selectively modified, have emerged as a potential hydrophilic component for the amphiphiles. Certain advantages associated with carbohydrate-based amphiphilic architectures such as their $\mathrm{pH}$ independent self-assembling behaviour, biocompatibility, and mildness to the skin, make them potential candidates for drug delivery applications. Owing to their excellent biocompatibility, they have been widely used as hydrophilic building blocks to construct amphiphilic structures. Moreover, carbohydrate's multiple hydroxyl groups are available for attachment of suitable fluorophores or targeting agents.

Our research group has been involved in the synthesis of advanced nanocarriers for drug delivery applications. We have synthesized numerous polymeric, ${ }^{50-52}$ dendritic $^{48}$ and small amphiphilic architectures ${ }^{53-57}$ for the encapsulation of various drugs and dyes. In one of our research works, water-soluble dendritic architectures having a symmetrical aromatic core, hydrophobic alkyl chain branches and glucose as hydrophilic surface groups were reported (Fig. 19). ${ }^{48}$ The self-assembly of these dendrimers, encapsulation of hydrophobic guest molecules and mechanism of cellular uptake was also studied. Alkyl chains of different lengths along with different types of hydrophilic groups were used to maintain proper hydrophilic-hydrophobic balance, and thus, to assist the formation of selfassembled aggregates in an aqueous medium. Of all the studied dendrimers, $\mathrm{C}_{6}$ alkylated dendrimers (19a and 19b) were found to form supramolecular nanostructures in water, where critical aggregation concentration (CAC) of G2 dendrimers were lower than that of G1 dendrimers. Also, the G2 dendrimers having a larger hydrophobic cavity exhibited higher encapsulation potential as compared to the smaller G1 dendrimers. The cellular uptake study of Nile red encapsulated dendrimer 19a employing A549 cell lines and using live cell imaging by confocal laser microscopy and flow cytometry at $4{ }^{\circ} \mathrm{C}$ and $37{ }^{\circ} \mathrm{C}$ revealed no significant change in uptake at the lower temperature $\left(4{ }^{\circ} \mathrm{C}\right)$, thus suggesting passive diffusion of
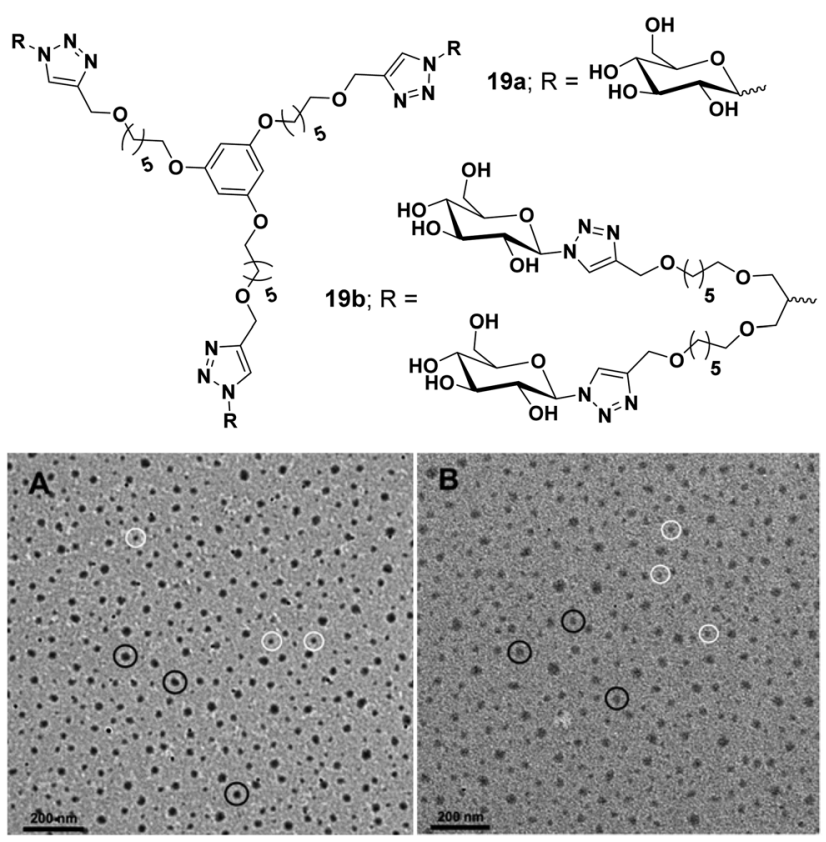

Fig. 19 Molecular structures and TEM images of amphiphiles (A) 19a and (B) 19b. Reprinted with permission from ref. 48. Copyright 2019 Royal Society of Chemistry.

dendrimer by non-endocytotic uptake mechanism, because the active uptake such as endocytosis is energy driven and should decrease with decrease in temperature.

In an another report, glucose- and galactose-based dimeric amphiphiles were synthesized that self-assembled into supramolecular architectures in an aqueous medium. ${ }^{49}$ Of all the three carbohydrate-based amphiphiles 20a-20c (Fig. 20), amphiphile 20b was fairly soluble in water and thus studied for encapsulation of hydrophobic guest molecules such as nimodipine, curcumin and Nile red. Cryo-TEM studies of the aqueous solutions of amphiphile 20b showed the formation of elongated threadlike aggregates. DLS measurement showed that, on moving from glucose-based amphiphile 20a to the lactose-based amphiphile 20c along with the increase in the alkyl chain length in the latter, the size decreased substantially from 156 to $36 \mathrm{~nm}$, respectively. A comparative study of the aggregates' diameter of amphiphiles 20a (156 nm) and 20b 

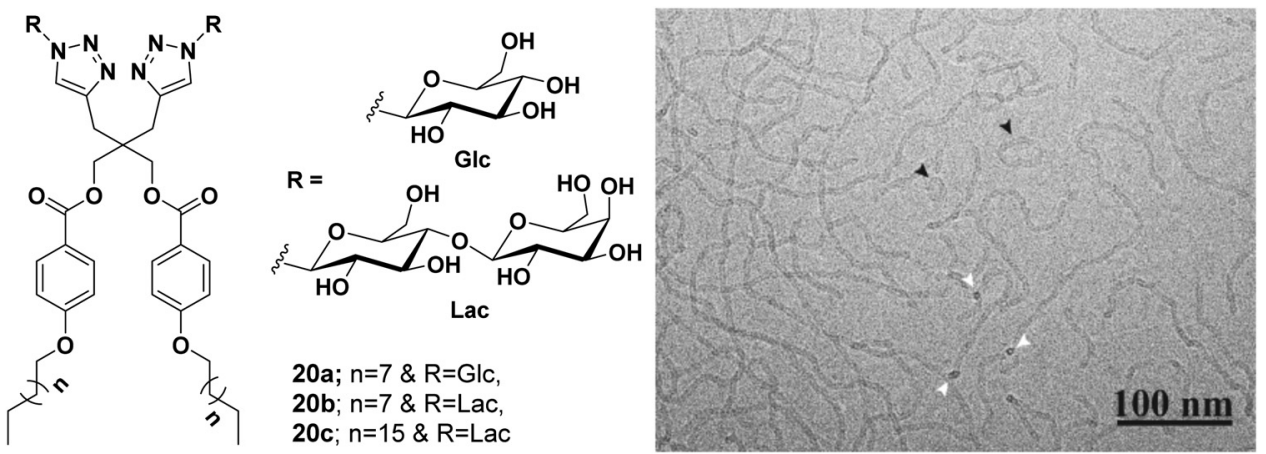

Fig. 20 Molecular structures of amphiphiles 20a-20c and cryo-TEM image of amphiphile 20b showing thread-like structures of uniform diameter $5.5 \pm 0.5 \mathrm{~nm}$, where circular cross-section is shown by white arrows. Some closed loop threads were also found (black arrow). Adapted with permission from ref. 49. Copyright 2018 Wiley-VCH Verlag GmbH \& Co. KGaA.

(40 nm) containing the same hydrophobic moiety but with an increased hydrophilic character in the latter, supplemented the fact that a balanced ratio of hydrophile and lipophile plays a prominent role in reducing the aggregate size.

Wang et al. reported the synthesis of sugar-based non-ionic modified Gemini amphiphiles 21a-21e by linking a spacer between the alkyl chains that led to the elimination of steric hindrance between bulky sugar moieties (Fig. 21).$^{58}$ The critical micelle concentration (CMC) values of these glycosylated Gemini amphiphiles were found to be lower than the monomeric counterparts and the conventional Gemini amphiphiles. Generally, the DLS size of aggregates increased with the concentration and the length of the attached alkyl chains. The size of the organization assemblies of Gemini alkylglucosides was found to be in the range of $25-400 \mathrm{~nm}$ at a concentration that was 5 times of CMC, and as the concentration increased by 25 fold, the hydrodynamic radius of the aggregate was observed in the range of $100-1000 \mathrm{~nm}$. The morphology of the aggregates was determined by TEM which indicated the formation of spherical and ellipsoidal vesicles. It was observed that as the concentration increased, all the amphiphiles formed vesicular aggregates except $\mathrm{C}_{14}$ glucoside (21c) which formed worm-like aggregates as shown in Fig. 21.

Meijer et al. employed various monosaccharides as versatile water solubilizing moieties to construct benzene-1,3,5-tricarboxamide (BTA)-based water-soluble architectures 22a-22h. ${ }^{59}$ Alkyl chain of different lengths (hexyl, octyl, decyl and dodecyl) and different hydrophilic saccharides ( $\beta$-glucose, $\alpha$-glucose, $\alpha$-galactose and $\alpha$-mannose) were used to maintain proper hydrophilic-hydrophobic balance (Fig. 22), and to assist the formation of self-assembled aggregates in an aqueous medium. Dodecyl and decyl spacers formed long fibers and small aggregates, respectively, while octyl and hexyl spacers gave molecularly dissolved BTAs, thus suggesting that the alkyl chain length influences the formation of different supramolecular self-assemblies. BTA core possessed strong interaction through $\pi-\pi$ stacking during the aggregate formation. It was observed that the introduction of different monosaccharides had little effect on the self-assembly behaviour of BTAs i.e. the presence of different monosaccharides showed similar self-assembly behaviour. This is in sharp contrast to the alkyl glycosides whose solubility and CMC strongly depend on the configuration of sugar, thus indicating that BTA based architectures do not simply behave as sugar-based amphiphiles. Furthermore, these BTA-based amphiphiles formed small aggregates at high temperature, which acted as a nucleus for elongation and turned into a long supramolecular assembly at moderate temperature, thus showing a high cooperative effect.

Further, the effect of glucose versus the tetraethylene glycol (TEG) unit as a water soluble peripheral skeleton in these amphiphiles was explored. ${ }^{60}$ From the FT-IR spectrum data, it was confirmed that both $22 \mathrm{a}$ and $\mathbf{2 2 h}$ amphiphiles formed stabilized supramolecular assemblies in water through hydrogen

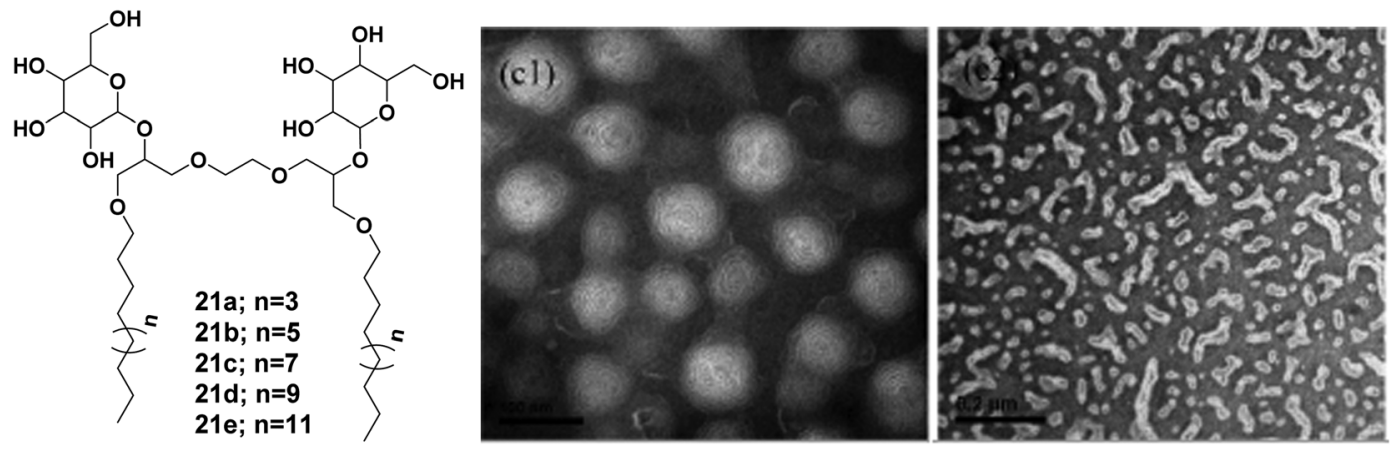

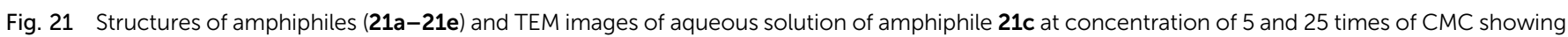
vesicles and worm-like nanostructures, respectively. Reprinted with permission from ref. 58 . Copyright 2013 American Chemical Society. 

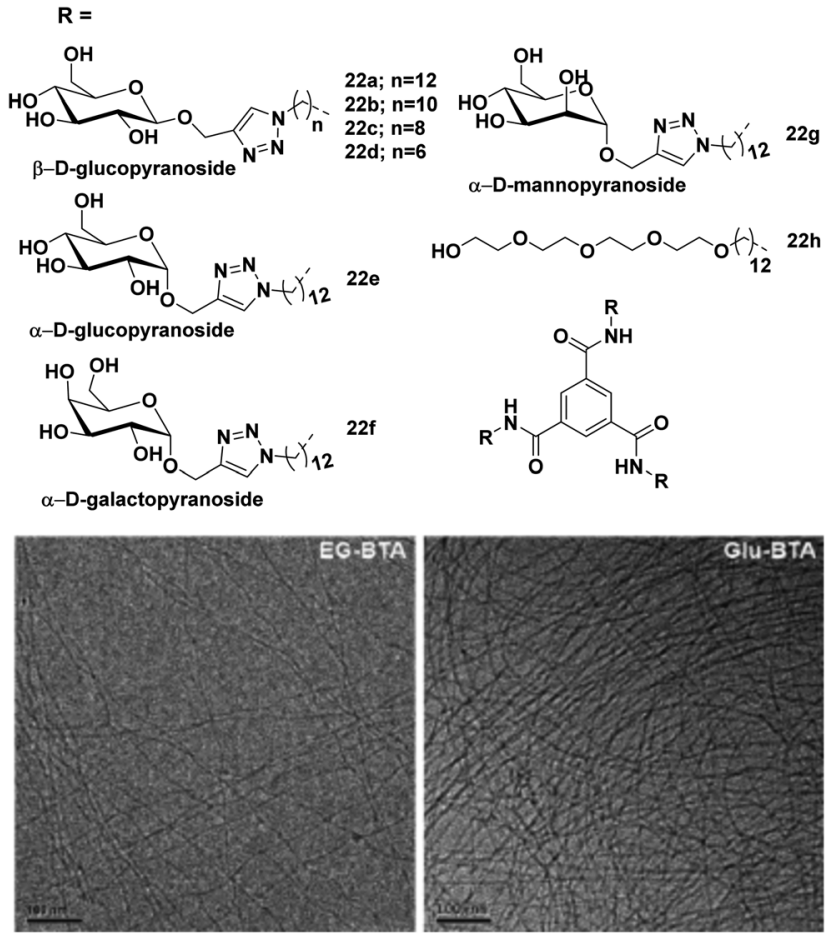

Fig. 22 Chemical structures of BTAs 22a-22h and cryo-TEM images of $\mathbf{2 2} \mathbf{h}$ and 22a in water. Scale bars are $100 \mathrm{~nm}$. Reprinted with permission from ref. 60. Copyright 2018 Royal Society of Chemistry.

bonding. Amphiphile 22a having numerous types of $\mathrm{H}$-bond donor and acceptor elements formed various $\mathrm{H}$-bonds with other glucose units within the polymer and with water. This increased the hydration of the polymer and changed its molecular dynamics. These additional $\mathrm{H}$-bonding interactions made the periphery of the supramolecular assembly more hydrated as compared to $\mathbf{2 2} \mathbf{h}$, and this was confirmed by molecular dynamic (MD) simulation. The Nile red encapsulation experiment also suggested that the core of amphiphile 22a was less hydrophobic than that of amphiphile $\mathbf{2 2 h}$ in supramolecular assemblies. These studies suggested the potential of the synthesized amphiphiles as a promising carrier for other hydrophobic dyes and drugs.

A similar approach was further extended to synthesize disaccharide ( $\beta$-cellobiose) and $\mathrm{OEG}_{4}$-mannose linked BTAs along with simple $\beta$-glucose and $\alpha$-mannose units as shown in Fig. 23. ${ }^{61}$ Monosaccharides such as glucose and mannose based amphiphiles self-assembled into long 1D fibbers of micrometer scale with chiral helicity, whereas cellobiose and $\mathrm{OEG}_{4}$-Man-based BTA molecules formed spherical micelles as evidenced by cryo-TEM (Fig. 23). Furthermore, the supramolecular copolymerization of amphiphiles BTA-Cel (23b) and BTA$\mathrm{OEG}_{4}$-Man (23d) was performed by mixing them in $2: 1,1: 1$ and $1: 2$ ratios. Co-polymerization of BTA-Glc (23a) and BTA-Cel (23b) resulted in the formation of fibrous nanostructures, and when BTA-Glc (23a) was co-polymerized with BTA-OEG 4 (23e), the formation of stable fiber structures was observed due to the proper alignment of cellobiose moieties. The optical activity of helical aggregate of BTA-Glc (23a) decreased with BTA-Cel (23b) and

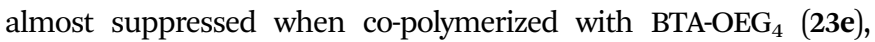
whereas BTA-Cel (23b) co-polymerized with BTA-OEG $_{4}$ (23e) showed an increase in optical activity. Hydrophobic pockets in co-assembled structures were further investigated by Nile red fluorescence assay, which indicated an increase in fluorescence intensity of the dye in the presence of co-assembled structures as compared to the Nile red in the water. Both homo and co-polymer showed similar results with the Nile red assay.
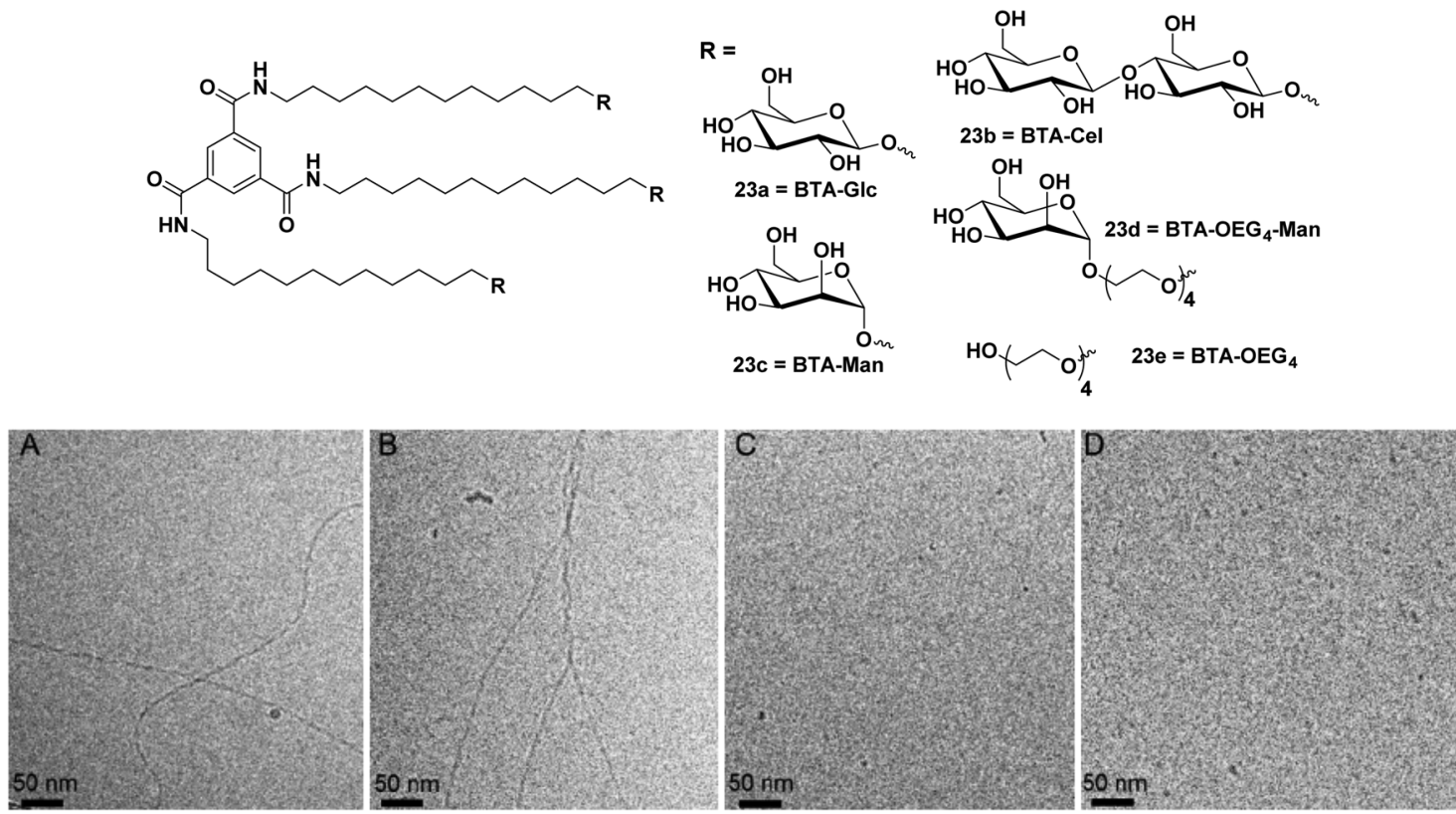

Fig. 23 Chemical structures of BTA amphiphiles 23a-23e and cryo-TEM images of (A) BTA-Glc (23a), (B) BTA-Man (23c), (C) BTA-Cel (23b), and (D) BTA-OEG 4 -Man (23d). Reprinted with permission from ref. 61. Copyright 2019 American Chemical Society. 


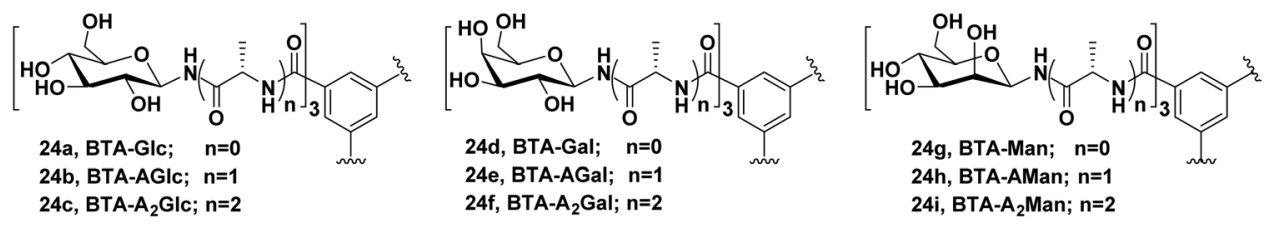

Fig. 24 Chemical structures of sugar-BTAs having different monosaccharides and varying length of alanine linkers.

Chen et al. synthesized three different categories of carbohydrate-coated BTA molecules 24a-24i as shown in Fig. 24 and studied their self-assembly behaviour. The effect of different surface groups (glucose, galactose and mannose) and different lengths of alanine linkers were investigated on the self-assembly of these BTAs. ${ }^{62}$ Direct attachment of monosaccharides to BTA core led to the formation of discrete selfassemblies, where BTA-Man (24g) self-assembled into twisted fiber nanostructures, while BTA-Glc (24a) and BTA-Gal (24d) formed vesicles and nanorings, respectively. With the introduction and growth of alanine linkers between BTA core and monosaccharides, irregularity in the self-assembly of these amphiphiles was observed, which concluded that the presence of alanine linker suppressed the interaction between monosaccharide and resulted into disordered aggregates. So, it was concluded that by employing different peptide linkers, different self-assembled nanostructures can be produced which can be used for different biomedical applications. These results point out the previously overlooked effect of peptide linkers in self-assembly, which could be useful in designing novel carbohydrate-based materials. Moreover, these architectures could act as potential drug carriers if incorporated with a hydrophobic alkyl chain between BTA and amino acids.

Geraci et al. investigated the self-assembly behaviour of calix[8]GlcNAc (25a) and calix[8]GlyGlcNAc (25b) (Fig. 25) at different $\mathrm{pH}$ and demonstrated their application as drug nanocarrier. ${ }^{63}$ The synthesized amphiphilic macrocycles selfassembled into stable vesicular aggregates in an aqueous solution that responded to $\mathrm{pH}$ change. The calix[8]GlcNAc and calix[8]GlyGlcNAc formed aggregates of average hydrodynamic radius of $110 \mathrm{~nm}$ and $200 \mathrm{~nm}$, respectively revealed by static and dynamic light scattering methods. In addition, the effect of $\mathrm{pH}$ on the aggregation behaviour was studied with

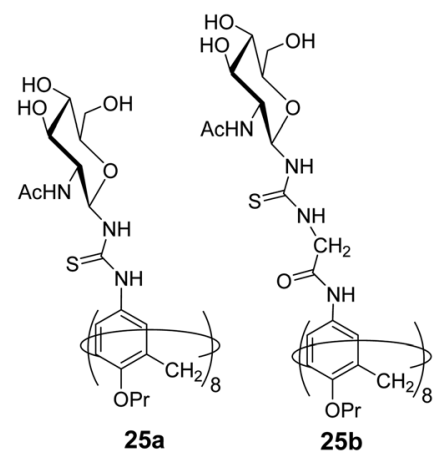

Fig. 25 Molecular structures of calix[8]GIcNAc (25a) and calix[8]GlyGlcNAc (25b).

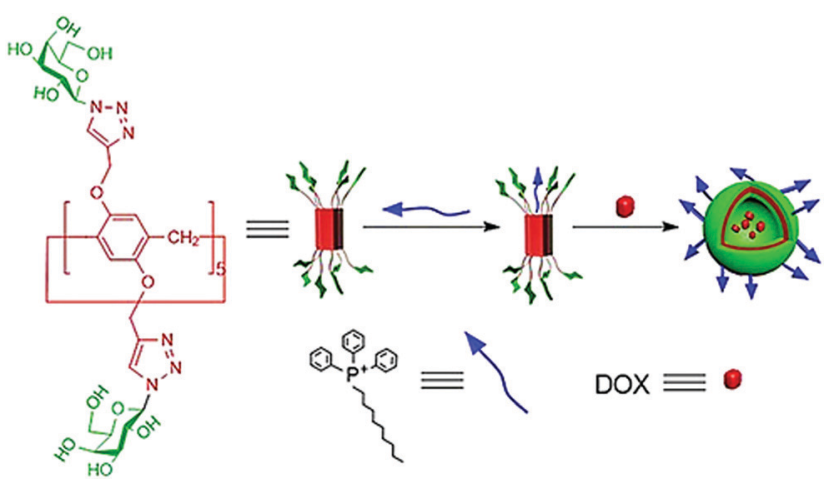

Fig. 26 Schematic representation of the dual-target supramolecular vesicles based on the host-guest complexation between galactose functionalized pillar[5]arene and D-TPP for targeting drug delivery. Reproduced with permission from ref. 64. Copyright 2018 Wiley-VCH Verlag GmbH \& Co. KGaA.

both nanostructures, as pH decreased from 7 to 3 , the diameter of the aggregate of both amphiphiles decreased to $5 \mathrm{~nm}$ with the transition in structure from vesicle to micelles. But as the $\mathrm{pH}$ increased in the range of 4.5-12 the size of the aggregates was found to be $150 \pm 50 \mathrm{~nm}$. Furthermore, the encapsulation of hydrophilic fluorescent dye rhodamine B in calix[8]GlcNAc molecule was also performed using fluorescence instrument and its release study was done which indicated that no leakage occurred at $\mathrm{pH} 7$ from the vesicles architecture, but as the $\mathrm{pH}$ of the solution decreased to 3 , the fluorescence intensity increased indicating the release of the dye in solution.

Pei et al. developed a supramolecular vesicle based on hostguest interaction between galactose containing pillar[5]arene and $n$-decyl triphenylphosphonium (D-TPP) as represented in Fig. $26 .^{64}$ The self-assembly of these amphiphiles showed vesicular nanostructures with an average diameter of $170 \mathrm{~nm}$. These supramolecular vesicles exhibited dual targeting potential due to cancer cell targeting ability caused by galactose unit and mitochondria targeting ability caused by TPP units of D-TPP. Doxorubicin loaded vesicles were helpful to improve drug accumulation into the mitochondria after entering the cancer cells. This work is a good example of the design and construction of dual-targeted supramolecular vesicles for drug delivery.

\section{Conclusion}

Carbohydrates, possessing excellent biodegradability, biocompatibility and large potential for chemical modifications, have emerged as promising candidates for designing amphiphilic architectures that are used in biological applications such as 
pathogen inhibition, lectin recognition and drug delivery. In addition to the general characteristics of carbohydrates, all carbohydrates have some specific intrinsic properties, which can be explored in the design of small non-ionic amphiphiles. Small amphiphiles have the tendency to self-assemble into well-defined nanostructures whose size, shape and morphology are related to the chemical structure of individual amphiphile. By varying the structure of amphiphile (hydrophilic surface carbohydrate group and hydrophobic tail), the structure of the self-assembled nanostructure can be controlled. In comparison to long polymeric or dendritic molecules, easy synthesis and modification of small amphiphiles make them potential candidates for the creation of self-assembled nanostructures for different biomedical applications. In this review, we have presented the ability of carbohydrate-based self-assembled nanostructures to act as a promising drug delivery candidate and to interact with specific lectin to inhibit pathogens. These results will be of interest to researchers working in this field to design advanced carbohydrate-based supramolecular architectures. Moreover, the obtained outcomes may also be useful in the future to modify and fine-tune the properties of carbohydratebased and other small amphiphiles and pave the way towards more sophisticated applications but not limited to drug delivery and pathogen inhibition.

Despite the progress made in the field of carbohydratebased amphiphilic nanostructures, only sporadic reports are available describing their interaction with the immune system. Several key issues including their toxicity and in vivo applicability and clearance also need to be addressed. There is a hope that in the near future, these self-assembled nanostructures will provide improved efficacy for pathogen inhibition and drug delivery to address ever increasing global requirements. We believe that future applications of carbohydrate-based amphiphiles will exceed our expectations and researchers across the globe will be able to work to make these amphiphiles the materials of choice as smart nanomedicines.

\section{Conflicts of interest}

There are no conflicts to declare.

\section{Acknowledgements}

We gratefully acknowledge the financial support from Biotechnology Industry Research Assistance Council (BIRAC), a Public Sector Enterprise, set up by the Department of Biotechnology (DBT), Government of India. Also, we are thankful to the University Grants Commission (UGC), India for providing Junior and Senior Research Fellowship to Ayushi Mittal.

\section{References}

1 A. Sorrenti, O. Illa and R. M. Ortuño, Chem. Soc. Rev., 2013, 42, 8200-8219.
2 G. M. Whitesides and B. Grzybowski, Science, 2002, 295, 2418.

3 M. Boncheva and G. M. Whitesides, MRS Bull., 2011, 30, 736-742.

4 V. Percec, G. Ungar and M. Peterca, Science, 2006, 313, 55. 5 J.-M. Lehn, Eur. Rev., 2009, 17, 263-280.

6 Rashmi, A. K. Singh, K. Achazi, S. Ehrmann, C. Böttcher, R. Haag and S. K. Sharma, Polym. Chem., 2020, 11, 6772.

7 P. Manchanda, K. Achazi, D. Verma, C. Böttcher, R. Haag and S. K. Sharma, Polymers, 2020, 12, 1421.

8 Parmanand, A. Mittal, A. K. Singh, Aarti, K. Achazi, C. Nie, R. Haagb and S. K. Sharma, RSC Adv., 2020, 10, 37555.

9 B. Parshad, S. Prasad, S. Bhatia, A. Mittal, Y. Pan, P. K. Mishra, S. K. Sharma and L. Fruk, RSC Adv., 2020, 10, 42098-42115.

10 D. Lombardo, M. A. Kiselev, S. Magazù and P. Calandra, Adv. Condens. Matter Phys., 2015, 2015.

11 L. C. Palmer and S. I. Stupp, Acc. Chem. Res., 2008, 41, 1674-1684.

12 X. Zhang and C. Wang, Chem. Soc. Rev., 2011, 40, 94-101.

13 M. Ramanathan, L. K. Shrestha, T. Mori, Q. Ji, J. P. Hill and K. Ariga, Phys. Chem. Chem. Phys., 2013, 15, 10580-10611.

14 H. Cui, M. J. Webber and S. I. Stupp, Pept. Sci., 2010, 94, 1-18.

15 J. Vonnemann, C. Sieben, C. Wolff, K. Ludwig, C. Böttcher, A. Herrmann and R. Haag, Nanoscale, 2014, 6, 2353-2360.

16 D.-W. Lee, T. Kim, I.-S. Park, Z. Huang and M. Lee, J. Am. Chem. Soc., 2012, 134, 14722-14725.

17 S. Bhatia, M. Hilsch, J. L. Cuellar-Camacho, K. Ludwig, C. Nie, B. Parshad, M. Wallert, S. Block, D. Lauster, C. Böttcher, A. Herrmann and R. Haag, Angew. Chem., Int. Ed., 2020, 59, 12417-12422.

18 C. Nie, M. Stadtmüller, H. Yang, Y. Xia, T. Wolff, C. Cheng and R. Haag, Nano Lett., 2020, 20, 5367-5375.

19 S. Bhatia, L. C. Camacho and R. Haag, J. Am. Chem. Soc., 2016, 138, 8654-8666.

20 C. Nie, B. Parshad, S. Bhatia, C. Cheng, M. Stadtmüller, A. Oehrl, Y. Kerkhoff, T. Wolff and R. Haag, Angew. Chem., Int. Ed., 2020, 59, 15532-15536.

21 C. Nie, M. Stadtmüller, B. Parshad, M. Wallert, V. Ahmadi, Y. Kerkhoff, S. Bhatia, S. Block, C. Cheng, T. Wolff and R. Haag, Sci. Adv., 2021, 7, eabd3803.

22 V. P. Torchilin, Nat. Rev. Drug Discovery, 2005, 4, 145-160.

23 S. Roy, K. Maiti, S. P. Moulik and R. Gosh, Colloids Surf., A, 2011, 377, 349.

24 W. Weis, J. H. Brown, S. Cusack, J. C. Paulson, J. J. Skehel and D. C. Wiley, Nature, 1988, 333, 426-431.

25 S. D. Knight and J. Bouckaert, in Glycoscience and Microbial Adhesion, ed. T. K. Lindhorst and S. Oscarson, Springer Berlin Heidelberg, Berlin, Heidelberg, 2009, pp. 67-107, DOI: 10.1007/128_2008_13.

26 N. Gilboa-Garber, Methods in Enzymology, Academic Press, 1982, vol. 83, pp. 378-385.

27 A. Bernardi, J. Jiménez-Barbero, A. Casnati, C. De Castro, T. Darbre, F. Fieschi, J. Finne, H. Funken, K.-E. Jaeger, M. Lahmann, T. K. Lindhorst, M. Marradi, P. Messner, 
A. Molinaro, P. V. Murphy, C. Nativi, S. Oscarson, S. Penadés, F. Peri, R. J. Pieters, O. Renaudet, J.-L. Reymond, B. Richichi, J. Rojo, F. Sansone, C. Schäffer, W. B. Turnbull, T. Velasco-Torrijos, S. Vidal, S. Vincent, T. Wennekes, H. Zuilhof and A. Imberty, Chem. Soc. Rev., 2013, 42, 4709-4727.

28 S. Bhatia, M. Dimde and R. Haag, MedChemComm, 2014, 5, 862-878.

29 A. G. Dal Bó, V. Soldi, F. C. Giacomelli, B. Jean, I. PignotPaintrand, R. Borsali and S. Fort, Soft Matter, 2011, 7, 3453-3461.

30 A. G. Dal Bó, V. Soldi, F. C. Giacomelli, C. Travelet, R. Borsali and S. Fort, Carbohydr. Res., 2014, 397, 31-36.

31 A. G. Dal Bó, V. Soldi, F. C. Giacomelli, C. Travelet, B. Jean, I. Pignot-Paintrand, R. Borsali and S. Fort, Langmuir, 2012, 28, 1418-1426.

32 B.-S. Kim, W.-Y. Yang, J.-H. Ryu, Y.-S. Yoo and M. Lee, Chem. Commun., 2005, 2035-2037, DOI: 10.1039/B419258C.

33 B.-S. Kim, D.-J. Hong, J. Bae and M. Lee, J. Am. Chem. Soc., 2005, 127, 16333-16337.

34 J.-H. Ryu, E. Lee, Y.-B. Lim and M. Lee, J. Am. Chem. Soc., 2007, 129, 4808-4814.

35 D.-W. Lee, T. Kim, I.-S. Park, Z. Huang and M. Lee, J. Am. Chem. Soc., 2012, 134, 14722-14725.

36 G. Na, Y. He, Y. Kim and M. Lee, Soft Matter, 2016, 12, 2846-2850.

37 K.-R. Wang, H.-W. An, L. Wu, J.-C. Zhang and X.-L. Li, Chem. Commun., 2012, 48, 5644-5646.

38 K.-R. Wang, H.-W. An, Y.-Q. Wang, J.-C. Zhang and X.-L. Li, Org. Biomol. Chem., 2013, 11, 1007-1012.

39 Y.-b. Lim, S. Park, E. Lee, J.-H. Ryu, Y.-R. Yoon, T.-H. Kim and M. Lee, Chem. - Asian J., 2007, 2, 1363-1369.

40 Y.-b. Lim, S. Park, E. Lee, H. Jeong, J.-H. Ryu, M. S. Lee and M. Lee, Biomacromolecules, 2007, 8, 1404-1408.

41 M. C. dos Santos, Y. M. S. Micheletto, N. P. da Silveira, L. da Silva Pinto, F. C. Giacomelli, V. R. de Lima, T. E. A. Frizon and A. G. Dal-Bó, Colloids Surf., B, 2016, 148, 12-18.

42 S. Zhang, R.-O. Moussodia, H.-J. Sun, P. Leowanawat, A. Muncan, C. D. Nusbaum, K. M. Chelling, P. A. Heiney, M. L. Klein, S. André, R. Roy, H.-J. Gabius and V. Percec, Angew. Chem., Int. Ed., 2014, 53, 10899-10903.

43 S. Zhang, R.-O. Moussodia, S. Vértesy, S. André, M. L. Klein, H.-J. Gabius and V. Percec, Proc. Natl. Acad. Sci. U. S. A., 2015, 112, 5585.

44 G. M. L. Consoli, F. Cunsolo, C. Geraci and V. Sgarlata, Org. Lett., 2004, 6, 4163-4166.
45 F. Sansone, L. Baldini, A. Casnati and R. Ungaro, Supramol. Chem., 2008, 20, 161-168.

46 S. Aleandri, A. Casnati, L. Fantuzzi, G. Mancini, G. Rispoli and F. Sansone, Org. Biomol. Chem., 2013, 11, 4811-4817.

47 G. Yu, Y. Ma, C. Han, Y. Yao, G. Tang, Z. Mao, C. Gao and F. Huang, J. Am. Chem. Soc., 2013, 135, 10310-10313.

48 B. Parshad, P. Yadav, Y. Kerkhoff, A. Mittal, K. Achazi, R. Haag and S. K. Sharma, New J. Chem., 2019, 43, 11984-11993.

49 S. Prasad, K. Achazi, B. Schade, R. Haag and S. K. Sharma, Macromol. Biosci., 2018, 18, 1800019.

50 M. Kumari, S. Gupta, K. Achazi, C. Böttcher, J. Khandare, S. K. Sharma and R. Haag, Macromol. Rapid Commun., 2015, 36, 254-261.

51 B. Parshad, M. Kumari, K. Achazi, C. Böttcher, R. Haag and S. Sharma, Polymers, 2016, 8, 311.

52 S. Kumar, K. Achazi, C. Böttcher, K. Licha, R. Haag and S. K. Sharma, Eur. Polym. J., 2015, 69, 416-428.

53 A. K. Singh, B. N. S. Thota, B. Schade, K. Achazi, A. Khan, C. Böttcher, S. K. Sharma and R. Haag, Chem. - Asian J., 2017, 12, 1796-1806.

54 Rashmi, A. K. Singh, K. Achazi, B. Schade, C. Böttcher, R. Haag and S. K. Sharma, RSC Adv., 2018, 8, 31777-31782.

55 A. Mittal, A. K. Singh, A. Kumar, Parmanand, K. Achazi, R. Haag and S. K. Sharma, Polym. Adv. Technol., 2020, 31, 1208-1217.

56 S. Prasad, K. Achazi, B. Schade, R. Haag and S. K. Sharma, Eur. Polym. J., 2018, 109, 506-522.

57 Krishna, B. Parshad, K. Achazi, C. Böttcher, R. Haag and S. K. Sharma, ChemMedChem, 2021, 16, 1457-1466.

58 S. Liu, R. Sang, S. Hong, Y. Cai and H. Wang, Langmuir, 2013, 29, 8511-8516.

59 C. M. A. Leenders, G. Jansen, M. M. M. Frissen, R. P. M. Lafleur, I. K. Voets, A. R. A. Palmans and E. W. Meijer, Chem. - Eur. J., 2016, 22, 4608-4615.

60 S. M. C. Schoenmakers, C. M. A. Leenders, R. P. M. Lafleur, X. Lou, E. W. Meijer, G. M. Pavan and A. R. A. Palmans, Chem. Commun., 2018, 54, 11128-11131.

61 S. I. S. Hendrikse, L. Su, T. P. Hogervorst, R. P. M. Lafleur, X. Lou, G. A. van der Marel, J. D. C. Codee and E. W. Meijer, J. Am. Chem. Soc., 2019, 141, 13877-13886.

62 J. Wang, W. Qi and G. Chen, Chin. Chem. Lett., 2019, 30, 587-591.

63 N. Micali, V. Villari, G. M. L. Consoli, F. Cunsolo and C. Geraci, Phys. Rev. E: Stat., Nonlinear, Soft Matter Phys., 2006, 73, 051904.

64 K. Shang, Y. Wang, Y. Lu, Z. Pei and Y. Pei, Isr. J. Chem., 2018, 58, 1241-1245. 